\title{
SMALL AMPLITUDE OSCILLATORY SHEAR PERMEATION FLOW OF CHOLESTERIC LIQUID CRYSTAL POLYMERS*
}

\author{
ZHENLU CUI
}

\begin{abstract}
We investigate the small amplitude oscillatory shear permeation flow (SAOSPF) of cholesteric liquid crystal polymers (CLCPs) using a mesoscopic model obtained from the Doi kinetic theory for flows of CLCPs. We model the system by Stokes hydrodynamic equations coupled with orientational dynamics and study the frequency-locked solutions of the system by employing a coarsegrained approach. The leading order solutions exhibit boundary layers of thickness of the order of the cholesteric pitch in low frequency regimes but in the order of $\frac{1}{\sqrt{\omega}}$ in high frequency regimes, where $\omega$ is the plate driven frequency. The response of CLCPs has been calculated. Viscoelastic behavior is only observed at intermediate frequencies and the material is essentially viscous at low and high frequency regimes. At both low and high frequencies, the real component of complex viscosity controls its magnitude which is of the order of the reciprocal of the cholesteric pitch at low frequencies while the order of 1 at high frequencies.
\end{abstract} ics.

Key words. Asymptotic expansion, cholesteric liquid crystals, permeation flows, hydrodynam-

AMS subject classifications. 76A15, 76M45.

\section{Introduction}

Cholesteric liquid crystals (CLCs) are mesophases between liquids and solids, in which the average molecular orientation exhibits a chiral (twisted) orientational pattern in a direction normal to the local averaged molecular orientation, which is known as the chiral helix [5, 12. Cholesterol esters, DNAs, colloidal suspensions of bacteriophages, and many biological materials are examples that can exhibit chiral phases under proper conditions. The chiral liquid crystal phase is delicate in that it can be sustained only under balanced conditions; otherwise, defects and disclinations can form easily. As a result, there are few quantitative experiments on the dynamics and rheology. The few available studies on flows of cholesterics are mostly based on Leslie-Ericksen (LE) continuum theory, which is remarkably successful in describing the dynamical behavior of low molecular-weight liquid crystals but not quite so for flows of liquid crystal polymers. Some studies based on perturbation techniques with the base helical axis in the flow direction [14, 15, 20, 16, 12, 15, 30, in the velocity gradient direction [19, 17, and in the vorticity direction in simple shear [13, 24, 25, 26] using the LE theory are available. Brief reviews on cholesteric rheology can be found in the books [12, 18.

Liquid crystals exhibit a viscoelastic response to an external stress. Coupling between the director and the velocity fields - known as backflow — leads to strongly non-Newtonian flow behavior. A particular striking example in flows of cholesterics is permeation where a cholesteric liquid crystal is subjected to an imposed flow in the direction of the helix but the helical structure remains nearly intact. The main feature of the permeation is its ultrahigh viscosity (by a factor of $\sim 10^{5}$ of pure nematic liquid crystals) in small shear. But, in strong shear, the helical structure is completely destroyed so that cholesterics behave essentially like pure nematic liquid crystals. An

\footnotetext{
${ }^{*}$ Received: December 30, 2009; accepted (in revised version): February 3, 2010. Communicated by Chun Liu.

${ }^{\dagger}$ The Department of Mathematics and Computer Science, Fayetteville State University, Fayetteville, NC 28301, USA (zcui@uncfsu.edu).
} 
explanation of this was first given by Helfrich [14, he argued that the usual parabolic profile is replaced by plug-like flow, with a constant velocity across the capillary (Poiseuille flow). De Gennes and Prost [12] added that this occurs over a length scale $\sim P$ where $P$ is the pitch of the helix. Experimental evidence of permeation flows is reported by Scaramuzza et al. in cholesterics subject to steplike strains in the direction of the helix 31. Prost et al. 23] conducted an stability study on permeation flows in 1 dimensionality order system. Rey carried out a series of studies on cholesteric liquid crystals based on LE theory to clarify and extend the previous studies [24, 25, 26, 27, 28, 29, 30. Marenduzzo et al. [21, 22] studied the sheared cholesterics numerically using a tensor model. They showed that the boundary anchoring condition as well as the gap width affect the flow and orientation structure in the shear cell. They also pointed out the importance of the secondary flow in their simulation [21, 22]. The authors [7] analytically studied permeation flows and resolved a long standing inconsistency in the study of chiral liquid crystal permeation flows.

Small amplitude oscillatory flows are a main rheological tool used to characterize viscoelasticity [1] in terms of the storage modulus $G^{\prime}$ and loss modulus $G^{\prime \prime}$. Previous work on small amplitude oscillatory shear of liquid crystals in a parallel plate geometry using Leslie-Ericksen theory has been presented for rod-like nematic liquid crystals [2, 9, 10, 11] and CLCs [27, 28, 29, 30]. Recently, the author [4] studied the linear viscoelastic response of nematic liquid crystal polymers to small amplitude oscillatory shear and pointed out rheological equivalence between shear flows with imposed oscillatory stress, velocity (or strain), and pressure. More recently, the author studied the linear viscoelasticity of CLCs using a tensor-based continuum theory 8. In this paper, we focus on small amplitude oscillatory permeation shear flow for CLCPs. The goal is to perform a systematic analysis of CLCPs subjected to small amplitude oscillatory shear permeation flow focusing on hydrodynamic analysis and the linear viscoelastic response. The paper is organized as follows. Section 2 presents the governing equations for flowing CLCPs. Section 3 investigates the hydrodynamics and the linear viscoelasticity of CLCPs under small amplitude oscillatory permeation flows. Section 4 gives the conclusions.

\section{Model formulation}

We begin with a brief review of the kinetic theory for cholesteric liquid crystal polymers (CLCPs) developed in 3 . We present the dimensionless governing system of equations along with the dimensionless parameter group only and refer readers to [3] for the detailed theory, the dimensional parameters, and the derivation.

We nondimensionalize the equations in the model using the characteristic length scale $h$ and the LCP relaxation time scale $t_{0}=t_{n}=\frac{1}{D_{r}^{0}}$, where $D_{r}^{0}$ is the rotary diffusivity, and denote the position vector by $\mathbf{x}$, the velocity vector by $\mathbf{v}$, the extra stress tensor by $\tau$, and the pressure by $p$, respectively. The dimensionless flow and stress variables are defined by:

$$
\tilde{\mathbf{v}}=\frac{t_{0}}{h} \mathbf{v}, \quad \tilde{\mathbf{x}}=\frac{1}{h} \mathbf{x}, \quad \tilde{t}=\frac{t}{t_{0}}, \quad \tilde{\tau}=\frac{h^{2}}{f_{0}} \tau, \quad \tilde{p}=\frac{h^{2}}{f_{0}} p,
$$

where $f_{0}=\rho h^{4} / t_{0}^{2}$ is an inertial force and $\rho$ is the CLCP density. Let $c$ be the CLCP number density, $k$ the Boltzmann constant, $T$ absolute temperature, $N$ a dimensionless concentration, $\eta$ the solvent viscosity, $\zeta_{i}, i=1,2,3$, three friction coefficients related to CLCP-solvent interaction, and $\mathcal{L}, L, L_{q}$ three length scales for the isotropic 
long-range interaction, anisotropic long-range interaction, and cholesteric length scale, respectively. The following 9 dimensionless parameters in the model arise:

$$
\begin{aligned}
& R e=\frac{\rho h^{2}}{t_{0} \eta}, \quad \alpha=\frac{3 c k T t_{0}^{2}}{h^{2} \rho}, \quad E r=\frac{8 h^{2}}{N D_{r}^{0} t_{0} \mathcal{L}^{2}}, \\
& \mu_{i}=\frac{3 c k T \zeta_{i} t_{0}}{h^{2} \rho}, \quad i=1,2,3, \quad \theta=\frac{L^{2}}{\mathcal{L}^{2}}, \theta_{q}=\frac{8 L_{q} h}{N \mathcal{L}^{2}} .
\end{aligned}
$$

$\alpha$ measures the strength of the elastic energy relative to that of the kinetic energy, $R e$ is the solvent Reynolds number, $E r$ is the Ericksen number which measures the strength of the short-range nematic potential relative to that of the isotropic distortional elastic energy, $\theta$ measures the strength of anisotropic distortional elasticity relative to the isotropic one whose range is limited to $[-1, \infty), 1 / \mu_{i}, i=1,2,3$, are three nematic Reynolds numbers, and $\theta_{q}$ parameterizes the chiral free energy relative to the isotropic distortional elastic one. We drop the tilde on all variables from now on so that all equations and figures in the following correspond to normalized (dimensionless) variables.

The dimensionless governing equations consisting of the continuity, momentum balance, orientation tensor equation and the constitutive equation for the extra stress tensor are given below.

Continuity equation

$$
\nabla \cdot \mathbf{v}=0
$$

Momentum balance equation

$$
\frac{d}{d t} \mathbf{v}=\nabla \cdot(-p \mathbf{I}+\tau)
$$

Orientation tensor equation

$$
\begin{aligned}
& \frac{d}{d t} \mathbf{M}-\Omega \cdot \mathbf{M}+\mathbf{M} \cdot \Omega-a[\mathbf{D} \cdot \mathbf{M}+\mathbf{M} \cdot \mathbf{D}] \\
= & -2 a \mathbf{D}: \mathbf{M}_{4}-6\left[\mathbf{Q}-N\left(\mathbf{M} \cdot \mathbf{M}-\mathbf{M}: \mathbf{M}_{4}\right)\right]+\frac{1}{E r}\left[\Delta \mathbf{M} \cdot \mathbf{M}+\mathbf{M} \cdot \Delta \mathbf{M}-2 \Delta \mathbf{M}: \mathbf{M}_{4}\right] \\
& +\frac{\theta}{2 E r}\left[(\nabla \nabla \mathbf{M}): \mathbf{M}_{4}+\left((\nabla \nabla \mathbf{M}) \vdots \mathbf{M}_{4}\right)^{T}+\mathbf{M}_{4}: \nabla \nabla \mathbf{M}+\left(\mathbf{M}_{4} \vdots \nabla \nabla \mathbf{M}\right)^{T}\right. \\
& \left.+\mathbf{M} \nabla \nabla \vdots \mathbf{M}_{4}+\left(\mathbf{M} \nabla \nabla \vdots \mathbf{M}_{4}\right)^{T}-4 \mathbf{M}_{6}:: \nabla \nabla \mathbf{M}-2 \mathbf{M}_{4} \nabla \nabla:: \mathbf{M}_{4}\right] \\
& -\frac{\theta_{q}}{E r}\left[\left(\mathbf{M}_{4, i k \beta j}+\mathbf{M}_{4, j k \beta i}\right) \mathbf{M}_{k \gamma, \mu} \epsilon_{\mu \gamma \beta}+\left(\mathbf{M}_{4, i k \alpha j}+\mathbf{M}_{4, j k \alpha i}\right) \mathbf{M}_{\alpha \gamma, \mu} \epsilon_{\mu \gamma k}\right. \\
& \left.-\left(\mathbf{M}_{\beta i} \mathbf{M}_{j \gamma, \mu}+\mathbf{M}_{\beta j} \mathbf{M}_{i \gamma, \mu}\right) \epsilon_{\mu \gamma \beta}-\mathbf{M}_{\alpha i} \mathbf{M}_{\alpha \gamma, \mu} \epsilon_{\mu \gamma j}-\mathbf{M}_{\alpha j} \mathbf{M}_{\alpha \gamma, \mu} \epsilon_{\mu \gamma i}\right],
\end{aligned}
$$

where $\mathbf{M}$ is $\mathbf{M}_{\mathbf{4}}$ and $\mathbf{M}_{\mathbf{6}}$ are the second, the fourth and sixth order moment of the orientational probability distribution function in the kinetic theory [33, respectively, $\mathbf{M}$ is positive definite symmetric matrix with trace $1, \Omega$ and $\mathbf{D}$ are the vorticity tensor and the rate of strain tensor, $N$ is the dimensionless concentration parameter, and $a=\frac{r^{2}-1}{r^{2}+1}$ parameterizes the aspect ratio $r$ of the spheroidal molecules $(0<a \leq 1$ corresponds to a rod-like molecule and $-1 \leq a<0$ for platelets [33]). The deviatoric part of $\mathbf{M}$

$$
\mathbf{Q}=\mathbf{M}-\mathbf{I} / 3
$$


is called the orientation tensor, which is the traceless normalization of the second moment $\mathbf{M}$. $\mathbf{Q}$ and $\mathbf{M}$ share an orthonormal frame of principal axes, called the directors in the nematic liquid literature. The symbols $\cdot,:,:,::$ denote the contraction operations on one pair, two pairs, three pairs, and four pairs of tensorial indices, respectively. More delicate index contractions are given explicitly for clarity in the equations whenever necessary.

Constitutive equation for the extra stress tensor

$$
\begin{aligned}
& \tau=\left(2 \eta+\mu_{3}(a)\right) \mathbf{D}+a \alpha\left[\mathbf{M}-\frac{\mathbf{I}}{3}-\frac{N}{2}\left(\left(\mathbf{I}+\frac{1}{3 N E r} \Delta\right) \mathbf{M} \cdot \mathbf{M}\right.\right. \\
& \left.\left.+\mathbf{M} \cdot\left(\mathbf{I}+\frac{1}{3 N E r} \Delta\right) \mathbf{M}-2\left(\mathbf{I}+\frac{1}{3 N E r} \Delta\right) \mathbf{M}: \mathbf{M}_{4}\right)\right] \\
& -\frac{\alpha}{6 E r}(\Delta \mathbf{M} \cdot \mathbf{M}-\mathbf{M} \cdot \Delta \mathbf{M})-\frac{\alpha}{12 E r}[\nabla \mathbf{M}: \nabla \mathbf{M}-(\nabla \nabla \mathbf{M}): \mathbf{M}] \\
& +\frac{a \alpha \theta}{12 E r}\left[4 \mathbf{M}_{6}:: \nabla \nabla \mathbf{M}+2 \mathbf{M}_{4} \nabla \nabla:: \mathbf{M}_{4}-\nabla \nabla \mathbf{M}: \mathbf{M}_{4}-\left(\nabla \nabla \mathbf{M}: \mathbf{M}_{4}\right)^{T}\right. \\
& \left.-\mathbf{M}_{4} \vdots \nabla \nabla \mathbf{M}-\left(\mathbf{M}_{4} \vdots \nabla \nabla \mathbf{M}\right)^{T}-\left(\mathbf{M} \nabla \nabla \vdots \mathbf{M}_{4}\right)^{T}-\mathbf{M} \nabla \nabla \vdots \mathbf{M}_{4}\right] \\
& -\frac{\alpha \theta}{12 E r}\left[\nabla \nabla \mathbf{M}: \mathbf{M}_{4}-\left(\nabla \nabla \mathbf{M}: \mathbf{M}_{4}\right)^{T}-\mathbf{M}_{4} \vdots \nabla \nabla \mathbf{M}+\left(\mathbf{M}_{4} \vdots \nabla \nabla \mathbf{M}\right)^{T}\right. \\
& \left.-\mathbf{M} \nabla \nabla: \mathbf{M}_{4}+\left(\mathbf{M} \nabla \nabla \vdots \mathbf{M}_{4}\right)^{T}\right]+\left[\mu_{1}(a)(\mathbf{D M}+\mathbf{M D})+\mu_{2}(a) \mathbf{D}: \mathbf{M}_{4}\right] \\
& -\frac{\alpha \theta_{q}}{3 E r}\left[\frac { a + 1 } { 2 } \left(\mathbf{M}_{j \beta} \mathbf{M}_{i \gamma, \mu} \epsilon_{\mu \gamma \beta}+\mathbf{M}_{\alpha j} \mathbf{M}_{\alpha \gamma, \mu} \epsilon_{\mu \gamma i}+\mathbf{M}_{j \gamma, \mu} \mathbf{M}_{i \beta} \epsilon_{\mu \gamma \beta}\right.\right. \\
& \left.+\mathbf{M}_{j \alpha, \mu} \mathbf{M}_{\alpha \beta} \epsilon_{\mu i \beta}\right)+\frac{a-1}{2}\left(\mathbf{M}_{i \beta} \mathbf{M}_{j \gamma, \mu} \epsilon_{\mu \gamma \beta}+\mathbf{M}_{\alpha i} \mathbf{M}_{\alpha \gamma, \mu} \epsilon_{\mu \gamma j}\right. \\
& \left.\left.\left.+\mathbf{M}_{j \gamma, \mu} \mathbf{M}_{\alpha \beta} \epsilon_{\mu i \beta}+\mathbf{M}_{j \alpha, \mu} \mathbf{M}_{\alpha \beta} \epsilon_{\mu i \beta}\right)-2 a \mathbf{M}_{4, i j \alpha \beta} \mathbf{M}_{\alpha \gamma, \mu} \epsilon_{\mu \gamma \beta}\right)\right] \text {. }
\end{aligned}
$$

Since the system is not closed, we would have to employ closure approximations on the higher moments. We use two simplest ones, quadratic and cubic closures, here. Namely, we approximate the fourth order tensor $\mathbf{M}_{4}$ using a tensor product of two second order tensors and the sixth order tensor $\mathbf{M}_{6}$ using a tensor product of three second order tensors :

$$
\mathbf{M}_{4}=\mathbf{M M}, \mathbf{M}_{6}=\mathbf{M M M} .
$$

In this approximation, the Frank elastic constants in the linearized limit are given by

$$
K_{1}=K_{2}=\frac{C}{E r} s_{u}^{2}\left(1+\theta\left(1-s_{u}\right) / 3\right), K_{3}=\frac{C}{E r} s_{u}^{2}\left(1+\theta\left(4 s_{u}+1\right) / 6\right),
$$

where $C$ is a parameter proportional to the concentration and $s_{u}$ is the equilibrium uniaxial order parameter [33]. For simplicity, in this paper we consider $\theta=0$, which corresponds to the single Frank constant approximation, i.e., $K_{1}=K_{2}=K_{3}$.

\section{CLCPs under SAOSPF}

We consider the permeation flow of CLCPs subjected to small amplitude oscillatory shear with the helix along the flow direction $z$, between two parallel plates at $x= \pm 1$ in Cartesian coordinates $(x, y, z)$ and moving with corresponding velocity $\mathbf{v}=(0,0, \pm \epsilon \cos \omega t)$, where $\epsilon=D e$ is the Deborah number defined by $D e=\frac{v_{0}}{D_{r}^{0} h}$ with 
$\mathrm{v}=\varepsilon \cos \omega \mathrm{t}$

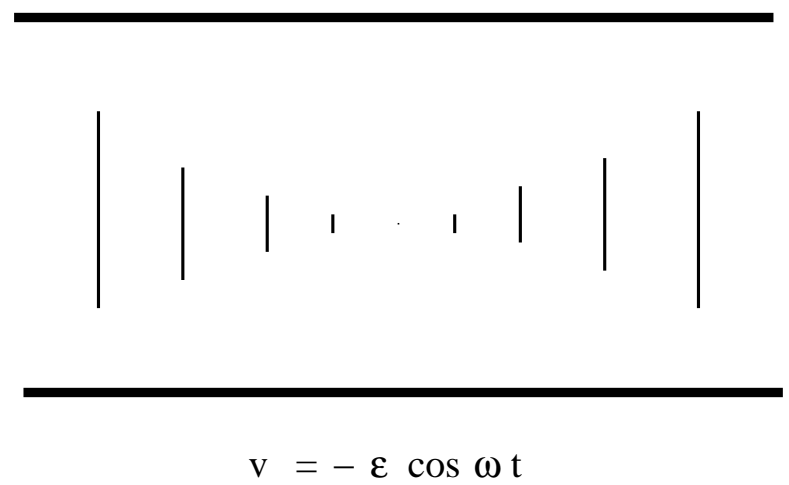

FIG. 3.1. The permeation flow geometry in a plane oscillatory cell. The gap width in the cell is 2. The CLC polymers in the cell is sheared by moving the upper plate with a speed $v=D e \cos \omega t$ and the lower one with $v=-D e \cos \omega t$. The helix of CLCPs is oriented along $z$-direction. At the bounding surfaces, the orientation tensor is assumed to equal to its equilibrium value.

the choice of $h$ the half gap width in the shear cell. The chiral nematic solution is found as 6

$$
\mathbf{Q}_{\mathbf{0}}=s_{0}\left(\mathbf{n}_{\mathbf{0}} \mathbf{n}_{\mathbf{0}}-\frac{\mathbf{I}}{3}\right), \mathbf{n}_{\mathbf{0}}=(\cos q z, \sin q z, 0)
$$

where $q$ and $s_{0}$ are determined by

$$
\begin{aligned}
& q=\frac{4 \theta_{q}\left(1-s_{0}\right)}{6+\theta\left(s_{0}+2\right)}, \\
& U\left(s_{0}\right)-\frac{8 \theta_{q}^{2}\left(2 s_{0}+1\right)\left[2+\left(2-s_{0}\right) \theta\right]\left(1-s_{0}\right)^{2}}{3 \operatorname{Er}\left[6+\theta\left(s_{0}+2\right)\right]^{2}} s_{0}^{2}=0, \\
& 0<s_{0}<1,
\end{aligned}
$$

where $U\left(s_{0}\right)=s_{0}\left[1-\frac{N}{3}\left(1-s_{0}\right)\left(2 s_{0}+1\right)\right]$.

Fig. 3.1 depicts the mechanism for the permeation oscillatory shear flow. When the small shear is imposed, $\mathbf{Q}$ is expected to exhibit biaxiality [32]. We parameterize the orientation tensor $\mathbf{Q}$ with three angle variables $\zeta, \xi$ and $\chi$, and two order parameters $s_{b}$ and $\beta_{b}$ :

$$
\mathbf{Q}=s_{b}\left(\mathbf{n n}-\frac{\mathbf{I}}{3}\right)+\beta_{b}\left(\mathbf{n}^{\perp} \mathbf{n}^{\perp}-\frac{\mathbf{I}}{3}\right),
$$

where

$$
\begin{aligned}
& \mathbf{n}=(\cos \zeta \cos \xi, \sin \zeta \cos \xi, \sin \xi), \\
& \mathbf{n}^{\perp}=(\sin \chi \sin \zeta-\cos \chi \sin \xi \cos \zeta,-\sin \chi \cos \zeta-\cos \chi \sin \xi \sin \zeta, \cos \chi \cos \xi),
\end{aligned}
$$

$\zeta$ is an in-plane tilt angle, $\xi$ and $\chi$ are out of plane tilt angles [32, 6, 7]. 
At the equilibrium, $\zeta_{0}=q z, \xi_{0}=0$, and $\chi=\chi_{0}$ (undefined). When $\mathbf{Q}$ is uniaxial, $\chi$ is irrelevant. We seek asymptotic solution in small Deborah number expansions:

$$
\begin{aligned}
s_{b}(x, z, t) & =s_{0}+\sum_{n=1}^{\infty} s_{n}(x, t) \epsilon^{n}, \\
\beta_{b}(x, z, t) & =\sum_{n=1}^{\infty} \beta_{n}(x, t) \epsilon^{n}, \\
(\zeta, \xi, \chi)(x, z, t) & =\left(q z+\sum_{n=1}^{\infty} \varphi_{n}(x, t) \epsilon^{n}, \sum_{n=1}^{\infty} \omega_{n}(x, t) \epsilon^{n}, \chi+\sum_{n=1}^{\infty} \psi_{n}(x, t) \epsilon^{n}\right), \\
\mathbf{v}(x, z, t) & =\left(0, \sum_{n=1}^{\infty} v_{y}^{(n)}(x, t) \epsilon^{n}, \sum_{n=1}^{\infty} v_{z}^{(n)}(x, t) \epsilon^{n}\right),
\end{aligned}
$$

where the secondary flow in $y$ direction is sought as well. The boundary conditions on the perturbation variables are

$$
\begin{aligned}
& \varphi_{n}( \pm 1)=\psi_{n}( \pm 1)=\omega_{n}( \pm 1)=s_{n}( \pm 1)=\beta_{n}( \pm 1)=v_{y}^{(n)}( \pm 1)=0 \\
& v_{z}^{(1)}( \pm 1)= \pm 1
\end{aligned}
$$

First, we expand the governing system of equations in powers of $\epsilon$ to obtain the asymptotic equations at each order of $O\left(\epsilon^{n}\right), n=0,1,2, \ldots$, in which we focus on the equations at order $O(\epsilon)$, the linearized equations. Secondly, we employ an ad hoc "coarse-grained" approximation, in which we average the linearized equations over a pitch of the cholesteric structure in $z$. We remark that the coarse-grain approximation is equivalent to the Galerkin projection onto the zeroth order Fourier space in the $z$ variable. Here we assume an out-of-plane flow along with an out-of-plane orientation structure. The coarse-grain linearized equations at order $O(\epsilon)$ are given in appendix A. The majority of the symbolic and numerical calculations presented in this paper were performed using the software MAPLE 12 by Waterloo Maple Inc. The values of parameters used in this paper are $N=6, E r=500, a=0.8, \alpha=1, \mu_{1}=0.007, \mu_{2}=$ $0.095, \mu_{3}=0.052, \theta_{q}=100.0$ and $\eta=0.001$.

Equations (A.1), (A.3), (A.4) and A.5) (see Appendix A) along with boundary conditions give $s_{1}(x, t)=\beta_{1}(x, t)=\omega(x, t)=0$ and $\chi$ is undetermined at this order. (A.6) automatically holds. We drop the subscript on $\varphi$ for brevity and use $v_{y}$ and $v_{z}$ to express $v_{y}^{(1)}$ and $v_{z}^{(1)}$. The linearized system reduces to

$$
\begin{aligned}
& \frac{\partial \varphi}{\partial t}+q v_{z}-\frac{1}{2} \frac{\partial v_{y}}{\partial x}-A_{1} \frac{\partial^{2} \varphi}{\partial x^{2}}=0, \\
& \frac{\partial^{2} v_{y}}{\partial x^{2}}-k_{1} \frac{\partial^{3} \varphi}{\partial x^{3}}=0 \\
& \frac{\partial^{2} v_{z}}{\partial x^{2}}-k_{2} q \frac{\partial^{2} \varphi}{\partial x^{2}}=0
\end{aligned}
$$

where

$$
\begin{aligned}
& A_{1}=\frac{2\left(s_{0}+2\right)}{3 E r} \\
& k_{1}=\frac{2 \alpha s_{0}^{2}}{\left(4 \mu_{1} s_{0}+3 \mu_{2} s_{0}^{2}+12 \eta+6 \mu_{3}\right) E r}, \\
& k_{2}=\frac{2 \alpha s_{0}{ }^{2}}{\left(6 \eta+3 \mu_{3}-\mu_{1} s_{0}\right) E r} .
\end{aligned}
$$


3.1. Hydrodynamics. Since the system (3.7) is linear and the plate driving conditions are sinusoidal in time, the standard analysis for determination of the linear viscoelastic moduli is to suppress transients and the total director, and the primary and secondary velocities are given by the sum of the following in-phase and out-ofphase components:

$$
\begin{aligned}
\varphi(x, t) & =\varphi_{1}(x) \cos \omega t+\varphi_{2}(x) \sin \omega t \\
v_{z}(x, t) & =u_{1}(x) \cos \omega t+u_{2}(x) \sin \omega t \\
v_{y}(x, t) & =v_{1}(x) \cos \omega t+v_{2}(x) \sin \omega t
\end{aligned}
$$

Note here and in the rest of the paper that in-phase means oscillation with the imposed Deborah number, and hence the in-phase temporal variation is cos $\omega t$, while the out of phase is sinwt. Substituting this ansatz into system (3.7), we obtain

$$
\begin{aligned}
\varphi_{1}(x)= & C_{1}[\cosh r x \sin s x-(\cosh r \sin s) x]+C_{2}[\sinh r x \cos s x-(\sinh r \cos s) x], \\
\varphi_{2}(x)=- & \frac{k_{2} q^{2}}{\omega}\left\{C_{1}[\cosh r x \sin s x-(\cosh r \sin s) x]+C_{2}[\sinh r x \cos s x-(\sinh r \cos s) x]\right\} \\
& +\frac{k_{1}}{\omega}\left[C_{1}\left(r^{2} \cosh r x \sin s x+2 r s \sinh r x \cos s x-s^{2} \cosh r x \sin s x\right)\right. \\
& \left.+C_{2}\left(r^{2} \sinh r x \cos s x-2 r s \cosh r x \sin s x-s^{2} \sinh r x \cos s x\right)\right]-q x, \\
u_{1}(x)= & k_{2} q C_{1}[\cosh r x \sin s x-(\cosh r \sin s) x] \\
& +k_{2} q C_{2}[\sinh r x \cos s x-(\sinh r \cos s) x]+x, \\
u_{2}(x)= & k_{2} q \varphi_{2}, \\
v_{1}=2(- & \left.A_{1}+k_{1}\right)\left[\left(r C_{1}-s C_{2}\right) \sinh r x \sin s x+\left(r C_{2}+s C_{1}\right) \cosh r x \cos s x\right] \\
& +2 A_{1}\left(C_{1} \cosh r \sin s+C_{2} \sinh r \cos s\right)+C_{3}, \\
& \omega \\
v_{2}=-\frac{s^{2}}{+} & r^{2}\left[2\left(-s C_{1}+r C_{2}\right) \cosh r x \cos s x+2\left(r C_{1}+s C_{2}\right) \sinh r x \sin s x\right. \\
& \left.-\left(s^{2}+r^{2}\right)\left(C_{1} \cosh r \sin s+C_{2} \sinh r \cos s\right) x^{2}\right]+\frac{k_{2} q^{2}}{\omega\left(s^{2}+r^{2}\right)}\left[\left(-2 k_{2} q^{2} r C_{1}\right.\right. \\
& \left.-2 k_{1} r^{2} s C_{2}+2 k_{1} r s^{2} C_{1}+2 k_{1} r^{3} C_{1}-2 k_{2} s q^{2} C_{2}-2 k_{1} s^{3} C_{2}\right) \sinh r x \sin s x \\
& +\left(-2 k_{2} q^{2} r C_{2}+2 k_{1} r^{2} s C_{1}+2 k_{1} r s^{2} C_{2}+2 k_{1} r^{3} C_{2}+2 k_{2} s q^{2} C_{1}\right. \\
& \left.+2 k_{1} s^{3} C_{1}\right) \cosh r x \cos s x+\left(k_{2} q C_{1} \cosh r \sin s+k_{2} q C_{2} \sinh r \cos s-1\right)\left(r^{2}\right. \\
& \left.\left.+s^{2}\right) q x^{2}\right]+\frac{A_{1}}{\omega}\left[\left(-k_{2} q^{2} r C_{1}+k_{2} q^{2} s C_{2}+k_{1} r^{3} C_{1}-3 k_{1} r s^{2} C_{1}-3 k_{1} s r^{2} C_{2}\right.\right. \\
& \left.+k_{1} s^{3} C_{2}\right) \sinh r x \sin s x+\left(-k_{2} q^{2} s C_{1}-k_{2} q^{2} r C_{2}+k_{1} r^{3} C_{2}-3 k_{1} r s^{2} C_{2}\right. \\
& \left.\left.+3 k_{1} s r^{2} C_{1}-k_{1} s^{3} C_{1}\right) \cosh r x \cos s x+k_{2} q^{2}\left(C_{1} \cosh r \sin s+C_{2} \sinh r \cos s\right)-q\right] \\
& +C_{4},
\end{aligned}
$$

where

$$
\begin{aligned}
& r=\sqrt[4]{\frac{k_{2}^{2} q^{4}+\omega^{2}}{k_{1}^{2}}} \cos \frac{\kappa}{2}, \\
& s=\sqrt[4]{\frac{k_{2}^{2} q^{4}+\omega^{2}}{k_{1}^{2}}} \sin \frac{\kappa}{2}, \\
& \kappa=\arctan \frac{\omega}{k_{2} q^{2}}
\end{aligned}
$$




\begin{tabular}{llllll}
\hline$\varphi_{1}$ & $\varphi_{2}$ & $u_{1}$ & $u_{2}$ & $v_{1}$ & $v_{2}$ \\
\hline odd & odd & odd & odd & even & even \\
\hline
\end{tabular}

TABLE 3.1. Symmetry properties of the frequency-locked solutions (3.10)
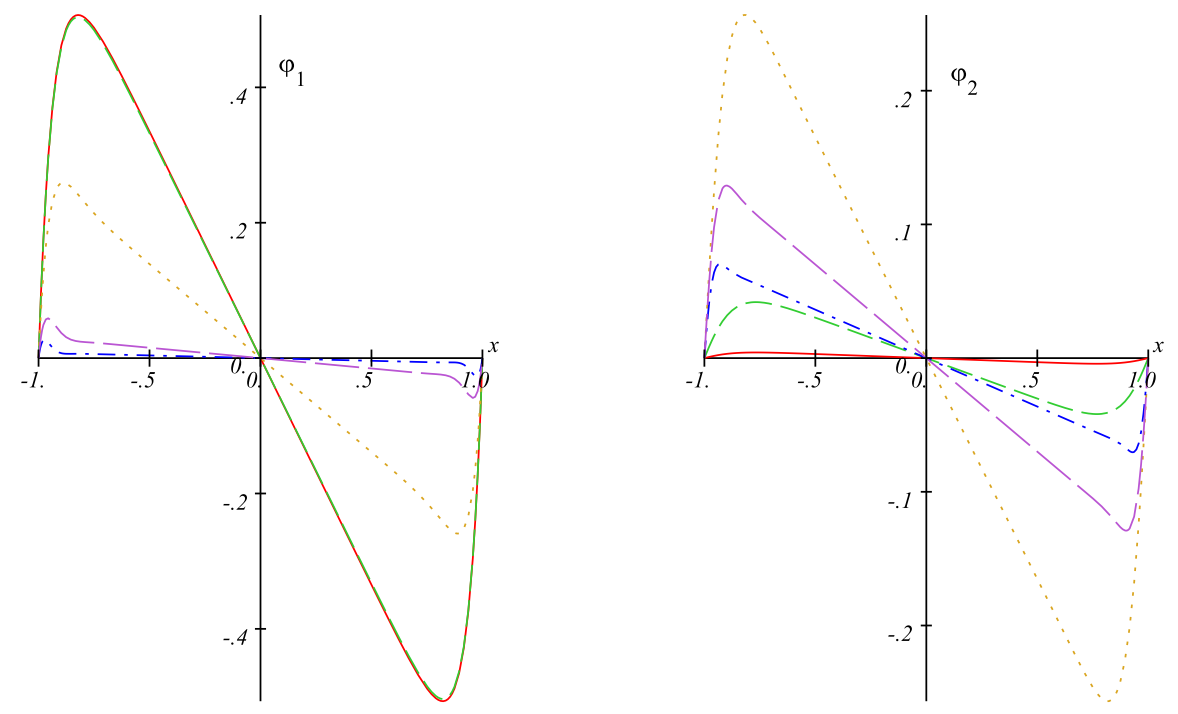

FIG. 3.2. The profiles of the director rotation components in phase (left) and out of phase (right) for rods $(a=0.8)$ at different frequencies $\omega=0.1$ (solid line), 1 (dashed line), 13 (dot), 50 (long-dashed line), and 100 (dash-dotted line).

and $C_{i}, \quad i=1,2,3,4$, are given in the Appendix B.

These frequency-locked solutions show symmetry properties under reflection $x \rightarrow$ $-x$, which are summarized in Table 3.1.

We note that the plate frequency induces a new length scale $\frac{1}{r}$, which defines a boundary layer near the plates for the solution system (3.10). In the high frequency regime, $r \sim \sqrt{\omega}$ while in the low frequency regime $r \sim q$. Thus the thickness of the boundary layer is proportional to $\frac{1}{\sqrt{\omega}}$ at high frequencies and $\frac{1}{q}$ at low frequencies. At the low frequency limit $(\omega \rightarrow 0)$, the steady permeation flow mode [6] is recovered. At high frequencies, the director is unable to relax sufficiently quickly to remain in phase with the imposed shear so that the out of phase components in the orientation tensor and flow velocities become significant. Fig. 3.2 represents in-phase and outof-phase components of the director as a function of the dimensionless gap width at five selected frequencies. The amplitude of the in-phase component decreases monotonically with the dimensionless frequency while the amplitude of the out-of-phase component increases and then decreases with the frequency. The maximum amplitude is achieved at $\omega \approx 13$ where the amplitudes of the in-phase component and the out-of-phase component are equal. As $\omega \rightarrow 0$ and $\omega \rightarrow \infty$, the amplitude of the outof-phase component will decrease to zero.

Fig. 3.3 represents the in-phase and out-of-phase components in the primary and secondary flow velocity at five selected frequencies. For the primary velocity, as $\omega \rightarrow 0$, 

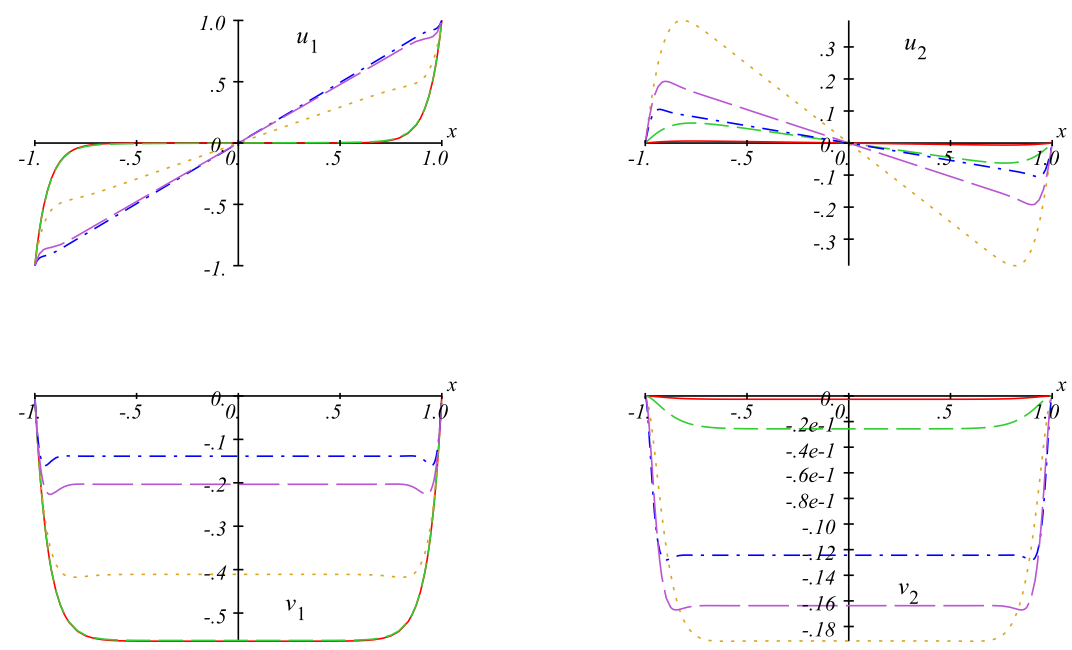

FIG. 3.3. The profiles of the primary flow (top) and the second flow (bottom) components in phase (left) and out of phase (right) for rods $(a=0.8)$ at different frequencies $\omega=0.1$ (solid line), 1 (dashed line), 13 (dot), 50 (long-dashed line), and 100 (dash-dotted line).

its in-phase component will go to the steady permeation modes and the out-of-phase component will go to zero. As $\omega$ increases, the in-phase component will be linear spanning the whole gap while the amplitude of the out-of-phase component increases and then decreases with the frequency, and achieves its maximum value around $\omega \approx$ 13. For the secondary velocity, both the in-phase and out of phase components are essentially constant away from the walls. The magnitude of the in phase component decreases monotonically with the dimensionless frequency while the amplitude of the out-of-phase component increases and then decreases with the frequency.

3.2. Linear viscoelasticity. Now we turn to linear viscoelastic analysis. The shear stress $\tau_{z x}^{*}=\tau_{0} \cos \omega t=\frac{6 \eta+3 \mu_{3}-\mu_{1} s_{0}}{6} \cos \omega t$. For steady shear flow, the dimensionless flow rate $F_{s}$ and the dimensionless apparent viscosity $\eta_{s}$ are given by the following relations [5, 7]:

$$
\begin{aligned}
\eta_{s} & =\frac{\tau_{s}}{2 F_{s}}, \\
F_{s} & =\int_{0}^{1}\left|v_{z}(x)\right| d x,
\end{aligned}
$$

where $\tau_{s}$ is the steady shear stress.

Generalizing these expressions for oscillatory shear flow using the dimensionless flow rate $F^{*}$ and the dimensionless complex viscosity $\eta^{*}$, we have

$$
\begin{aligned}
& \eta^{*}=\frac{\tau_{z x}^{*}}{2 F^{*}}, \\
& F^{*}=F_{i} \cos \omega t+F_{o} \sin \omega t, \\
& \eta^{*}=\eta^{\prime}-i \eta^{\prime \prime}
\end{aligned}
$$

where $F_{i}=\int_{0}^{1}\left|u_{1}(x)\right| d x$ and $F_{o}=\int_{0}^{1}\left|u_{2}(x)\right| d x$ are in phase and out of phase flow rate respectively, and they are found as 


$$
\begin{aligned}
F_{i}= & -\frac{1}{2\left(r^{2}+s^{2}\right)}\left[-2 k_{2} q C_{2} \sinh (r) \sin (s) s-2 k_{2} q C_{1} \sinh (r) r \sin (s)\right. \\
& +2 k_{2} q C_{1} \cosh (r) \cos (s) s-2 k_{2} q C_{2} \cosh (r) r \cos (s)-s^{2}-r^{2} \\
& +k_{2} q C_{2} n \sinh (r) r^{2} \cos (s)+k_{2} q C_{2} \sinh (r) \cos (s) s^{2}+q k_{2} C_{1} \cosh (r) \sin (s) r^{2} \\
& \left.+q k_{2} C_{1} \cosh (r) \sin (s) s^{2}-2 k_{2} q C_{1} s+2 k_{2} q C_{2} r\right], \\
F_{o}= & \frac{k_{2} q}{2 w\left(r^{2}+s^{2}\right)}\left[2 s^{3} k_{1} C_{1}+2 k_{1} C_{2} r^{3}+2 r k_{1} C_{2} s^{2}+2 k_{2} q^{2} s C_{1}+2 k_{1} C_{1} r^{2} s\right. \\
& -2 k_{2} q^{2} r C_{2}-2 r k_{1} C_{1} s^{2} \sinh (r) \sin (s)-2 k_{1} C_{2} r^{3} \cosh (r) \cos (s) \\
& +2 k_{2} q^{2} s C_{2} \sinh (r) \sin (s)+q r^{2}+q s^{2}+2 s^{3} k_{1} C_{2} \sinh (r) \sin (s) \\
& +2 k_{2} q^{2} r C_{1} \sinh (r) \sin (s)+2 k_{2} q^{2} r C_{2} \cosh (r) \cos (s)-2 s^{3} k_{1} C_{1} \cosh (r) \cos (s) \\
& +2 k_{1} C_{2} r^{2} s \sinh (r) \sin (s)-k_{2} q^{2} C_{2} \sinh (r) \cos (s) r^{2}-2 r k_{1} C_{2} s^{2} \cosh (r) \cos (s) \\
& -2 k_{1} C_{1} r^{3} \sinh (r) \sin (s)-k_{2} q^{2} C_{1} \cosh (r) \sin (s) s^{2}-k_{2} q^{2} C_{1} \cosh (r) \sin (s) r^{2} \\
& -2 k_{2} q^{2} s C_{1} \cosh (r) \cos (s)-2 k_{1} C_{1} r^{2} s \cosh (r) \cos (s) \\
& \left.-k_{2} q^{2} C_{2} \sinh (r) \cos (s) s^{2}\right] . \\
&
\end{aligned}
$$

Separating real and imaginary components in 3.13$)_{1}$ (the in phase component corresponds to the real part while the out of phase one corresponds to the imaginary part), we find

$$
\begin{aligned}
& \eta^{\prime} F_{i}+\eta^{\prime \prime} F_{o}=\frac{\tau_{0}}{2}, \\
& \eta^{\prime} F_{o}-\eta^{\prime \prime} F_{i}=0 .
\end{aligned}
$$

One can solve this system easily to obtain

$$
\begin{gathered}
\eta^{\prime}=\frac{\tau_{0}}{2} \frac{F_{i}}{F_{i}^{2}+F_{o}^{2}}, \\
\eta^{\prime \prime}=\frac{\tau_{0}}{2} \frac{F_{o}}{F_{i}^{2}+F_{o}^{2}} .
\end{gathered}
$$

In rheology, the fluid response may be characterized by the viscoelastic functions, $\eta^{\prime}, \eta^{\prime \prime}$, the storage modulus $G^{\prime}=\eta^{\prime \prime} \omega$, and the loss modulus $G^{\prime \prime}=\eta^{\prime} \omega$ [1]. Fig. 3.4 depicts the typical $G^{\prime \prime}$ and $G^{\prime}$ computed numerically as a function of the dimensionless frequency. These predictions are consistent with the previous work on CLCs by Rey [27, 30]. 


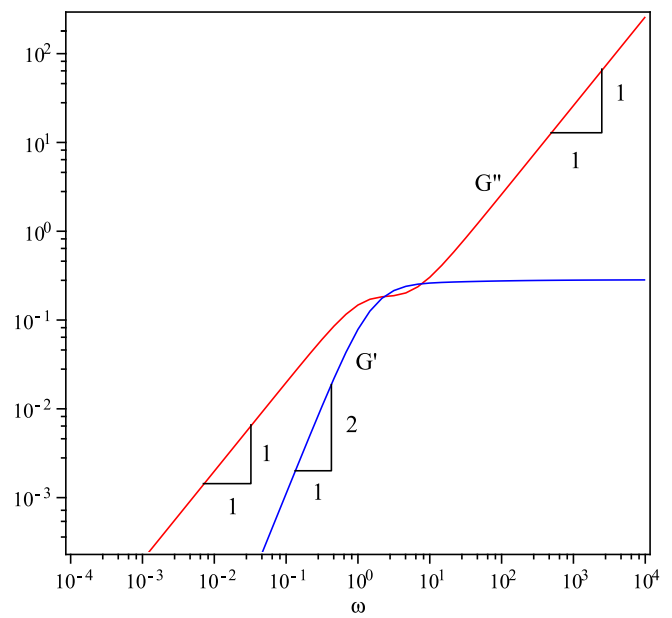

FIG. 3.4. The computed $G^{\prime}$ and $G^{\prime \prime}$ as a function of the dimensionless frequency $\omega$. At low frequencies, the slopes for $G^{\prime}$ and $G^{\prime \prime}$ are 2 and 1 , respectively. At high frequencies, the slopes of $G^{\prime}$ and $G^{\prime \prime}$ are 0 and 1 , respectively.

At low frequencies,

$$
\begin{aligned}
G^{\prime} \approx & 1 / 2\left(2 q \sqrt{k_{1}} \sqrt{k_{2}}\left(\cosh \left(\frac{\sqrt{k_{2}} q}{\sqrt{k_{1}}}\right)\right)^{3}-6 k_{1}\left(\cosh \left(\frac{\sqrt{k_{2}} q}{\sqrt{k_{1}}}\right)\right)^{3}\right. \\
& +4 q \sqrt{k_{2}} \sqrt{k_{1}}\left(\cosh \left(\frac{\sqrt{k_{2}} q}{\sqrt{k_{1}}}\right)\right)^{2}-6 k_{1}\left(\cosh \left(\frac{\sqrt{k_{2}} q}{\sqrt{k_{1}}}\right)\right)^{2} \sinh \left(\frac{\sqrt{k_{2}} q}{\sqrt{k_{1}}}\right) \\
& +2 q \sqrt{k_{1}} \sqrt{k_{2}}\left(\cosh \left(\frac{\sqrt{k_{2}} q}{\sqrt{k_{1}}}\right)\right)^{2} \sinh \left(\frac{\sqrt{k_{2}} q}{\sqrt{k_{1}}}\right)+6 k_{1} \cosh \left(\frac{\sqrt{k_{2}} q}{\sqrt{k_{1}}}\right) \\
& -q \sqrt{k_{1}} \sqrt{k_{2}} \cosh \left(\frac{\sqrt{k_{2}} q}{\sqrt{k_{1}}}\right)+4 q \sqrt{k_{2}} \sqrt{k_{1}} \cosh \left(\frac{\sqrt{k_{2}} q}{\sqrt{k_{1}}}\right) \sinh \left(\frac{\sqrt{k_{2}} q}{\sqrt{k_{1}}}\right) \\
& \left.+3 k_{1} \sinh \left(\frac{\sqrt{k_{2}} q}{\sqrt{k_{1}}}\right)-2 q \sqrt{k_{2}} \sqrt{k_{1}}\right)\left(\cosh \left(\frac{\sqrt{k_{2}} q}{\sqrt{k_{1}}}\right)+\sinh \left(\frac{\sqrt{k_{2}} q}{\sqrt{k_{1}}}\right)+1\right) \\
& \frac{1}{\sqrt{k_{2}}} q^{-1}\left(4\left(\cosh \left(\frac{\sqrt{k_{2}} q}{\sqrt{k_{1}}}\right)\right)^{4}+4\left(\cosh \left(\frac{\sqrt{k_{2}} q}{\sqrt{k_{1}}}\right)\right)^{3} \sinh \left(\frac{\sqrt{k_{2}} q}{\sqrt{k_{1}}}\right)\right. \\
& -2\left(\cosh \left(\frac{\sqrt{k_{2}} q}{\sqrt{k_{1}}}\right)\right)^{3}-5\left(\cosh \left(\frac{\sqrt{k_{2}} q}{\sqrt{k_{1}}}\right)\right)^{2} \\
& -2\left(\cosh \left(\frac{\sqrt{k_{2}} q}{\sqrt{k_{1}}}\right)\right)^{2} \sinh \left(\frac{\sqrt{k_{2}} q}{\sqrt{k_{1}}}\right)+2 \cosh \left(\frac{\sqrt{k_{2}} q}{\sqrt{k_{1}}}\right) \\
& \left.-3 \cosh \left(\frac{\sqrt{k_{2}} q}{\sqrt{k_{1}}}\right) \sinh \left(\frac{\sqrt{k_{2}} q}{\sqrt{k_{1}}}\right)+\sinh \left(\frac{\sqrt{k_{2}} q}{\sqrt{k_{1}}}\right)+1\right)^{-1} k_{1}^{-3 / 2} \omega^{2}=O(E r) \omega^{2}
\end{aligned}
$$




$$
\begin{aligned}
& G^{\prime \prime} \approx \frac{1}{4} q \sqrt{k_{2}}\left(\left(\cosh \left(\frac{q \sqrt{k_{2}}}{\sqrt{k_{1}}}\right)+\sinh \left(\frac{q \sqrt{k_{2}}}{\sqrt{k_{1}}}\right)\right)^{2}-1\right)^{2} \\
& \frac{1}{\sqrt{k_{1}}}\left(-2\left(\cosh \left(\frac{q \sqrt{k_{2}}}{\sqrt{k_{1}}}\right)\right)^{2}-\cosh \left(\frac{q \sqrt{k_{2}}}{\sqrt{k_{1}}}\right) \sinh \left(\frac{q \sqrt{k_{2}}}{\sqrt{k_{1}}}\right)+\sinh \left(\frac{q \sqrt{k_{2}}}{\sqrt{k_{1}}}\right)\right. \\
&-2\left(\cosh \left(\frac{q \sqrt{k_{2}}}{\sqrt{k_{1}}}\right)\right)^{3}+2 \cosh \left(\frac{q \sqrt{k_{2}}}{\sqrt{k_{1}}}\right) \\
& \quad-2\left(\cosh \left(\frac{q \sqrt{k_{2}}}{\sqrt{k_{1}}}\right)\right)^{2} \sinh \left(\frac{q \sqrt{k_{2}}}{\sqrt{k_{1}}}\right)+2\left(\cosh \left(\frac{q \sqrt{k_{2}}}{\sqrt{k_{1}}}\right)\right)^{4} \\
&\left.+2\left(\cosh \left(\frac{q \sqrt{k_{2}}}{\sqrt{k_{1}}}\right)\right)^{3} \sinh \left(\frac{q \sqrt{k_{2}}}{\sqrt{k_{1}}}\right)\right)^{-1} \omega=O(q) \omega
\end{aligned}
$$

The last expressions in $G^{\prime}$ and $G^{\prime \prime}$ are based on $q \gg 1$.

At high frequencies,

$$
\begin{aligned}
& G^{\prime} \approx O\left(\frac{q^{2}}{E r}\right), \\
& G^{\prime \prime} \approx O(\omega),
\end{aligned}
$$

which show that $G^{\prime}$ is independent of $\omega$ while $G^{\prime \prime}$ is independent of $q$ and $E r$.

Another important material function is the phase angle between stress and strain or loss angle [1] defined as

$$
\delta=\arctan \frac{G^{\prime \prime}}{G^{\prime}} .
$$

Fig. 3.5 depicts the loss angle as a function of the dimensionless frequency shows that CLCPs behave as viscous fluid at low and high frequency $(\delta=\pi / 2)$ and in between they behave as a viscoelastic materials. By solving $\frac{d \delta}{d \omega}=0$, we obtain the resonance frequency at which the maximum elastic storage occurs is $\omega=4.25$.

Fig. 3.6 depicts the magnitude of the complex viscosity $\left(\eta^{*}\right)$ and the components of the complex viscosity $\eta^{\prime}$ and $\eta^{\prime \prime}$ as a function of the dimensionless frequency $\omega$. The magnitude of the complex viscosity and component $\eta^{\prime}$ display three regions, with low and high frequency plateaus and a power-law intermediate region. For both low and high frequencies, $\left|\eta^{*}\right| \rightarrow \eta^{\prime}$, which shows that the fluid response of these limits are Newtonian like. At low frequency regime, $\eta^{\prime}=O(q)$ while at high frequency regime, $\eta^{\prime}=O(1) . \eta^{\prime \prime}$ achieves its maxima at $\omega \approx 1.0$ which shows that at the intermediate frequency regime, the fluid is viscoelastic in the oscillatory response. 


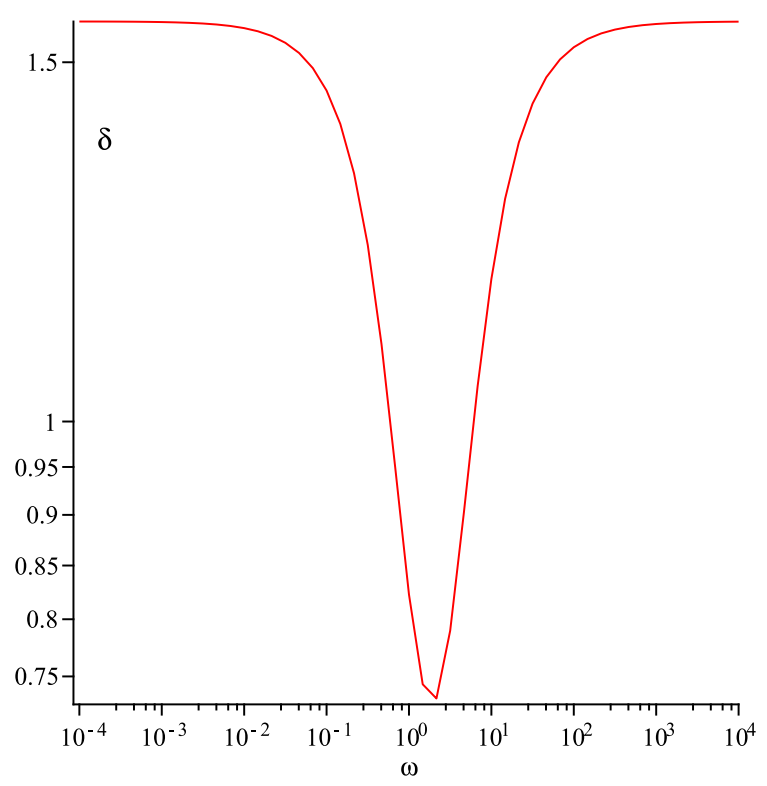

FIG. 3.5. The profile of the loss angle as a function of the dimensionless frequency. It shows that viscoelasticity is only observed at intermediate frequencies and the material is essentially viscous at low and high frequency regimes.

\section{Conclusion}

In summary, we have derived the governing equations for the permeation flow in CLCPs subject to a small amplitude oscillatory shear and studied the director dynamics and flow behavior as well as linear viscoelasticity. Small amplitude oscillatory permeation shear flow of CLCPs is characterized by a Newtonian response at low and high frequencies and viscoelasticity only exists in intermediate frequency regime. At low frequencies, the steady permeation modes are recovered and the director rotates in phase with the applied shear. At high frequencies, the out of phase component dominates the dynamics. The asymptotic formulas for the loss modulus $\left(G^{\prime \prime}\right)$ and storage modulus $\left(G^{\prime}\right)$ are obtained at both low and high frequencies. In the low frequency limit, both the loss modulus and storage modulus exhibit a classical frequency $\omega$ dependence but their magnitudes are of order $O(E r)$ and $O(q)$, respectively. The magnitudes of complex viscosity are calculated, which displays three regimes, with two Newtonian plateaus at low and high frequencies while a power-law response at intermediate frequency regime. These results are consistent with those obtained from a continuum model [8] and they are insensitive to a molecular shape. 


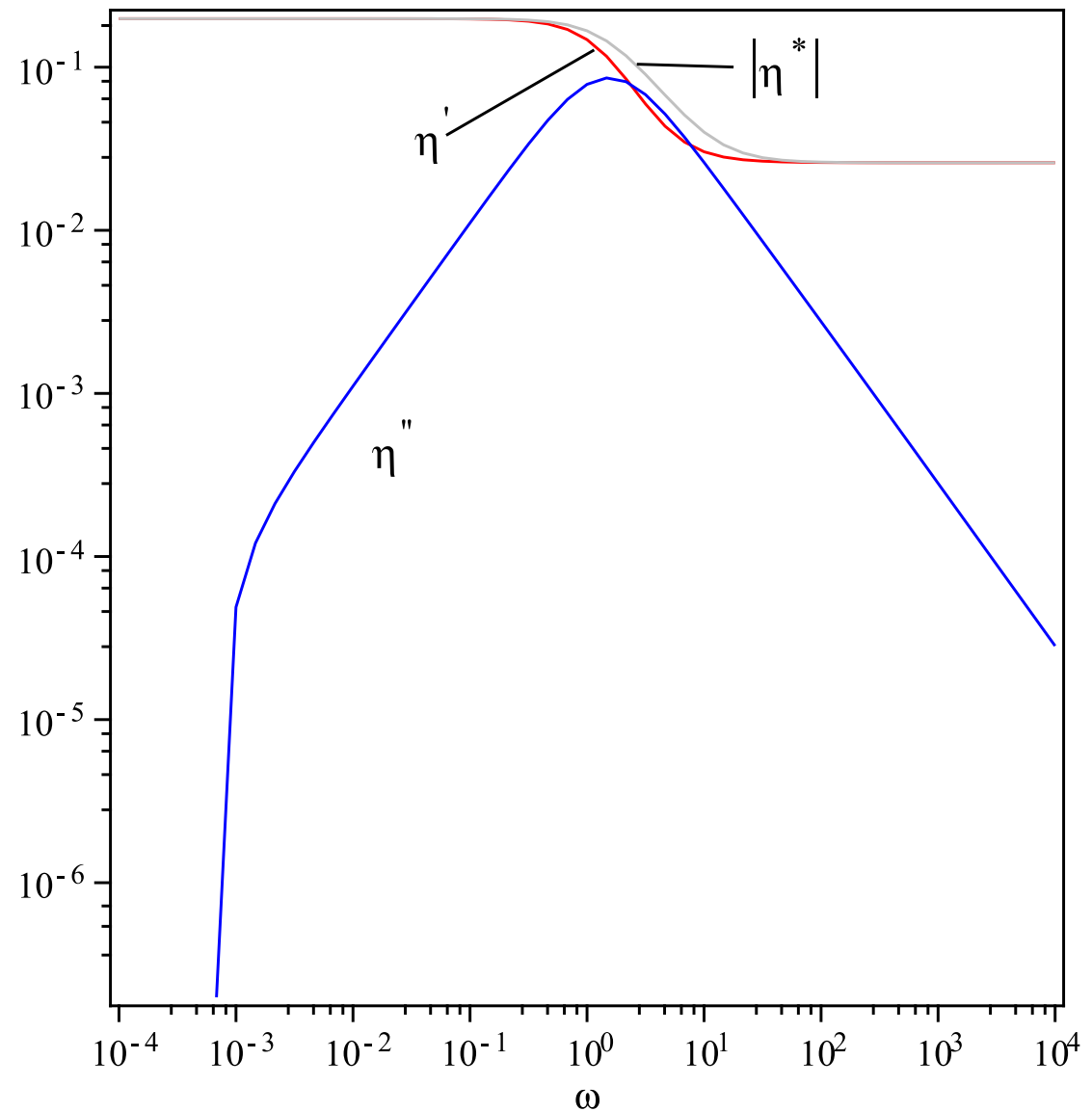

FIG. 3.6. The magnitude of the complex viscosity $\left(\eta^{*}\right)$ and the components of complex viscosity as a function of the dimensionless frequency $(\omega)$. At both low and high frequencies, the magnitude of the complex viscosity is dominated by its real component. 


\section{Appendix A. The governing equations at order $O(\epsilon)$.}

$$
\begin{aligned}
& 12 s_{0}^{2}\left(\frac{\partial}{\partial x} \omega\right) q-48 s_{0}^{3}\left(\frac{\partial}{\partial x} \omega\right) q-36 \operatorname{Er} N s_{0}{ }^{3} \beta_{1}\left(\cos \left(\chi_{1}\right)\right)^{2} \pi \\
& +54 \operatorname{Er} N s_{0} \beta_{1}\left(\cos \left(\chi_{1}\right)\right)^{2} \pi+24 s_{0}^{2} \operatorname{Er} N \beta_{1} \pi-9\left(\frac{\partial}{\partial t} \beta_{1}\right) \operatorname{Er} s_{0}\left(\cos \left(\chi_{1}\right)\right)^{2} \pi \\
& -18 \beta_{1} \operatorname{Er} N\left(\cos \left(\chi_{1}\right)\right)^{2} \pi+6 s_{0} \operatorname{Er} N s_{1} \pi+12 s_{0} q^{2} \beta_{1}\left(\cos \left(\chi_{1}\right)\right)^{2} \pi \\
& -54 E r s_{0} \beta_{1}\left(\cos \left(\chi_{1}\right)\right)^{2} \pi+3\left(\frac{\partial}{\partial t} s_{1}\right) E r s_{0} \pi+18 s_{1} E r s_{0} \pi \\
& +3\left(\frac{\partial}{\partial t} \beta_{1}\right) \operatorname{Er} s_{0} \pi+9\left(\frac{\partial}{\partial t} \beta_{1}\right) \operatorname{Er}\left(\cos \left(\chi_{1}\right)\right)^{2} \pi+6 s_{0}^{2} q^{2} s_{1} \pi \\
& +6 s_{1} \operatorname{Er} N \pi+12\left(\frac{\partial^{2}}{\partial x^{2}} \beta_{1}\right)\left(\cos \left(\chi_{1}\right)\right)^{2} s_{0} \pi-6\left(\frac{\partial^{2}}{\partial x^{2}} \beta_{1}\right)\left(\cos \left(\chi_{1}\right)\right)^{2} s_{0}{ }^{2} \pi-12 s_{0} q^{2} \beta_{1} \pi \\
& +54 \beta_{1} \operatorname{Er}\left(\cos \left(\chi_{1}\right)\right)^{2} \pi+6 \beta_{1} \operatorname{Er} N \pi-36 s_{0}^{3} q^{2} s_{1} \pi+18 \beta_{1} \operatorname{Er} s_{0} \pi \\
& -18 s_{0}^{2} q^{2} \beta_{1} \pi+12 s_{0}^{3} q^{2} \beta_{1} \pi+12 s_{0} q^{2} s_{1} \pi+2\left(\frac{\partial^{2}}{\partial x^{2}} s_{1}\right) \pi \\
& +12 s_{0}{ }^{3} q^{2} \beta_{1}\left(\cos \left(\chi_{1}\right)\right)^{2} \pi-48 s_{0}^{2} \operatorname{Er} N s_{1} \pi-18 s_{1} \operatorname{Er} \pi-18 \beta_{1} \operatorname{Er} \pi+4\left(\frac{\partial^{2}}{\partial x^{2}} s_{1}\right) s_{0}{ }^{3} \pi \\
& -6\left(\frac{\partial^{2}}{\partial x^{2}} s_{1}\right) s_{0}^{2} \pi+6\left(\frac{\partial^{2}}{\partial x^{2}} \beta_{1}\right) s_{0}^{2} \pi-3\left(\frac{\partial}{\partial t} s_{1}\right) \operatorname{Er} \pi-3\left(\frac{\partial}{\partial t} \beta_{1}\right) \operatorname{Er} \pi x \\
& -6\left(\frac{\partial^{2}}{\partial x^{2}} \beta_{1}\right)\left(\cos \left(\chi_{1}\right)\right)^{2} \pi-2\left(\frac{\partial^{2}}{\partial x^{2}} \beta_{1}\right) s_{0}{ }^{3} \pi \\
& -6\left(\frac{\partial^{2}}{\partial x^{2}} \beta_{1}\right) s_{0} \pi+2\left(\frac{\partial^{2}}{\partial x^{2}} \beta_{1}\right) \pi-30 s_{0} \operatorname{Er} N \beta_{1} \pi \\
& +36 s_{0}{ }^{3} \operatorname{Er} N s_{1} \pi+30 s_{0}{ }^{2} q^{2} \beta_{1}\left(\cos \left(\chi_{1}\right)\right)^{2} \pi=0 .
\end{aligned}
$$

$$
\begin{aligned}
& -60 s_{0}\left(\frac{\partial^{2}}{\partial x^{2}} \varphi\right) \pi+540 s_{0}{ }^{3} \operatorname{Er} N \varphi \pi-90 v_{z} s_{0}{ }^{2} q \operatorname{Er} \pi+90 v_{z} s_{0} q \operatorname{Er} \pi-360 s_{0}{ }^{4} \operatorname{Er} N \varphi \pi \\
& -180 \operatorname{Er} N s_{0} \varphi \pi+30 s_{0}^{2}\left(\frac{\partial^{2}}{\partial x^{2}} \varphi\right) \pi+30 s_{0}{ }^{3}\left(\frac{\partial^{2}}{\partial x^{2}} \varphi\right) \pi+80 q\left(\frac{\partial}{\partial x} \beta_{1}\right) \sin \left(\chi_{1}\right) \cos \left(\chi_{1}\right) \\
& +44 q s_{0}\left(\frac{\partial}{\partial x} \beta_{1}\right) \sin \left(\chi_{1}\right) \cos \left(\chi_{1}\right)+128 q s_{0}^{2}\left(\frac{\partial}{\partial x} \beta_{1}\right) \sin \left(\chi_{1}\right) \cos \left(\chi_{1}\right)
\end{aligned}
$$

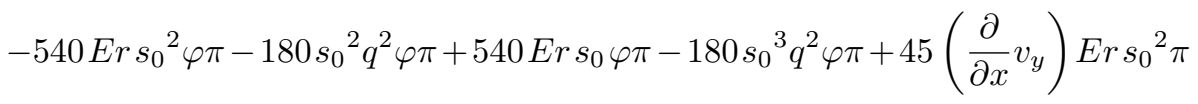

$$
\begin{aligned}
& +360 s_{0}{ }^{4} q^{2} \varphi \pi+90 s_{0}\left(\frac{\partial}{\partial t} \varphi\right) \operatorname{Er} \pi-45\left(\frac{\partial}{\partial x} v_{y}\right) \operatorname{Er} s_{0} \pi-90 s_{0}{ }^{2}\left(\frac{\partial}{\partial t} \varphi\right) \operatorname{Er} \pi=0 .
\end{aligned}
$$




$$
\begin{aligned}
& -12 s_{0}\left(\frac{\partial^{2}}{\partial x^{2}} \omega\right) \pi+6 s_{0}^{3}\left(\frac{\partial^{2}}{\partial x^{2}} \omega\right) \pi+6 s_{0}^{2}\left(\frac{\partial^{2}}{\partial x^{2}} \omega\right) \pi+4 q s_{0} \frac{\partial}{\partial x} s_{1}+9 s_{0}^{2} q^{2} \omega \pi \\
& +72 s_{0}{ }^{4} q^{2} \omega \pi+18 s_{0}\left(\frac{\partial}{\partial t} \omega\right) \operatorname{Er} \pi+108 s_{0} \omega \operatorname{Er} \pi-54 s_{0}^{3} q^{2} \omega \pi+16 q s_{0}{ }^{2} \frac{\partial}{\partial x} s_{1} \\
& -108 s_{0}^{2} \omega E r \pi+108 s_{0}^{3} \operatorname{Er} N \omega \pi-72 s_{0}{ }^{4} \operatorname{Er} N \omega \pi-18 s_{0}{ }^{2}\left(\frac{\partial}{\partial t} \omega\right) \operatorname{Er} \pi \\
& -36 s_{0} \omega \operatorname{Er} N \pi+16 q \frac{\partial}{\partial x} s_{1}+16 q\left(\frac{\partial}{\partial x} \beta_{1}\right)\left(\cos \left(\chi_{1}\right)\right)^{2}-20 q s_{0}\left(\frac{\partial}{\partial x} \beta_{1}\right)\left(\cos \left(\chi_{1}\right)\right)^{2} \\
& -32 q s_{0}{ }^{2}\left(\frac{\partial}{\partial x} \beta_{1}\right)\left(\cos \left(\chi_{1}\right)\right)^{2}+8 q s_{0} \frac{\partial}{\partial x} \beta_{1}+8 q s_{0}{ }^{2} \frac{\partial}{\partial x} \beta_{1}-16 q \frac{\partial}{\partial x} \beta_{1}=0 .
\end{aligned}
$$

$$
\begin{aligned}
& 36 s_{0}^{2}\left(\frac{\partial}{\partial x} \omega\right) q-48 s_{0}^{3}\left(\frac{\partial}{\partial x} \omega\right) q-36 \operatorname{Er} N s_{0}^{3} \beta_{1}\left(\cos \left(\chi_{1}\right)\right)^{2} \pi \\
& +54 \operatorname{Er} N s_{0} \beta_{1}\left(\cos \left(\chi_{1}\right)\right)^{2} \pi+30 s_{0}^{2} q^{2} \beta_{1}\left(\cos \left(\chi_{1}\right)\right)^{2} \pi+24 s_{0}{ }^{2} \operatorname{Er} N \beta_{1} \pi \\
& -9\left(\frac{\partial}{\partial t} \beta_{1}\right) \operatorname{Ers}_{0}\left(\cos \left(\chi_{1}\right)\right)^{2} \pi-18 \beta_{1} \operatorname{Er} N\left(\cos \left(\chi_{1}\right)\right)^{2} \pi+6 s_{0} \operatorname{Er} N s_{1} \pi \\
& +12 s_{0} q^{2} \beta_{1}(x, t)\left(\cos \left(\chi_{1}\right)\right)^{2} \pi-54 \operatorname{Er} s_{0} \beta_{1}\left(\cos \left(\chi_{1}\right)\right)^{2} \pi \\
& +3\left(\frac{\partial}{\partial t} s_{1}\right) E r s_{0} \pi+18 s_{1} E r s_{0} \pi+3\left(\frac{\partial}{\partial t} \beta_{1}\right) E r s_{0} \pi \\
& +9\left(\frac{\partial}{\partial t} \beta_{1}\right) \operatorname{Er}\left(\cos \left(\chi_{1}\right)\right)^{2} \pi+6 s_{0}^{2} q^{2} s_{1} \pi+6 s_{1} \operatorname{Er} N \pi \\
& +12\left(\frac{\partial^{2}}{\partial x^{2}} \beta_{1}\right)\left(\cos \left(\chi_{1}\right)\right)^{2} s_{0} \pi-6\left(\frac{\partial^{2}}{\partial x^{2}} \beta_{1}\right)\left(\cos \left(\chi_{1}\right)\right)^{2} s_{0}{ }^{2} \pi-12 s_{0} q^{2} \beta_{1} \pi \\
& +54 \beta_{1} \operatorname{Er}\left(\cos \left(\chi_{1}\right)\right)^{2} \pi+6 \beta_{1} \operatorname{Er} N \pi-36 s_{0}{ }^{3} q^{2} s_{1} \pi+18 \beta_{1} \operatorname{Er} s_{0} \pi-18 s_{0}{ }^{2} q^{2} \beta_{1} \pi \\
& +12 s_{0}^{3} q^{2} \beta_{1} \pi+12 s_{0} q^{2} s_{1} \pi+2\left(\frac{\partial^{2}}{\partial x^{2}} s_{1}\right) \pi+12 s_{0}^{3} q^{2} \beta_{1}\left(\cos \left(\chi_{1}\right)\right)^{2} \pi \\
& -48 s_{0}^{2} \operatorname{Er} N s_{1} \pi-18 s_{1} \operatorname{Er} \pi-18 \beta_{1} \operatorname{Er} \pi+4\left(\frac{\partial^{2}}{\partial x^{2}} s_{1}\right) s_{0}{ }^{3} \pi \\
& -6\left(\frac{\partial^{2}}{\partial x^{2}} s_{1}\right) s_{0}{ }^{2} \pi+6\left(\frac{\partial^{2}}{\partial x^{2}} \beta_{1}\right) s_{0}^{2} \pi-3\left(\frac{\partial}{\partial t} s_{1}\right) \operatorname{Er} \pi-3\left(\frac{\partial}{\partial t} \beta_{1}\right) \operatorname{Er} \pi \\
& -6\left(\frac{\partial^{2}}{\partial x^{2}} \beta_{1}\right)\left(\cos \left(\chi_{1}\right)\right)^{2} \pi-2\left(\frac{\partial^{2}}{\partial x^{2}} \beta_{1}\right) s_{0}^{3} \pi-6\left(\frac{\partial^{2}}{\partial x^{2}} \beta_{1}\right) s_{0} \pi \\
& +2\left(\frac{\partial^{2}}{\partial x^{2}} \beta_{1}\right) \pi-30 s_{0} \operatorname{Er} N \beta_{1} \pi+36 s_{0}^{3} \operatorname{Er} N s_{1} \pi+48 s_{0}\left(\frac{\partial}{\partial x} \omega\right) q=0 \text {. }
\end{aligned}
$$




$$
\begin{aligned}
& 3 q s_{0}\left(\frac{\partial}{\partial x} \beta_{1}\right)\left(\cos \left(\chi_{1}\right)\right)^{2} \pi+4 q\left(\frac{\partial}{\partial x} s_{1}\right) \pi+4 q\left(\frac{\partial}{\partial x} \beta_{1}\right) \pi-16 s_{0} \frac{\partial^{2}}{\partial x^{2}} \omega \\
& -96 s_{0}{ }^{4} \operatorname{Er} N \omega+8 s_{0}{ }^{3} \frac{\partial^{2}}{\partial x^{2}} \omega+144 s_{0}^{3} \operatorname{Er} N \omega-48 s_{0} \omega \operatorname{Er} N+24 s_{0}\left(\frac{\partial}{\partial t} \omega\right) E r \\
& +12 q^{2} s_{0}{ }^{2} \omega+96 s_{0}{ }^{4} q^{2} \omega-72 s_{0}{ }^{3} q^{2} \omega+8 s_{0}{ }^{2} \frac{\partial^{2}}{\partial x^{2}} \omega-2 q s_{0}\left(\frac{\partial}{\partial x} \beta_{1}\right) \pi \\
& +4 q s_{0}{ }^{2}\left(\frac{\partial}{\partial x} s_{1}\right) \pi-2 q s_{0}{ }^{2}\left(\frac{\partial}{\partial x} \beta_{1}\right) \pi-12 q\left(\frac{\partial}{\partial x} \beta_{1}\right)\left(\cos \left(\chi_{1}\right)\right)^{2} \pi+q s_{0}\left(\frac{\partial}{\partial x} s_{1}\right) \pi \\
& -24 s_{0}{ }^{2}\left(\frac{\partial}{\partial t} \omega\right) E r+144 s_{0} \omega E r-144 s_{0}{ }^{2} \omega E r=0 .
\end{aligned}
$$

$$
\begin{aligned}
& 6\left(\frac{\partial^{3}}{\partial x^{3}} s_{1}\right) s_{0}^{2} \pi+90 q^{3} s_{0}^{2} \omega-3\left(\frac{\partial^{3}}{\partial x^{3}} \beta_{1}\right) s_{0}{ }^{2} \pi-36 q^{3} s_{0}{ }^{3} \omega-36 s_{0}{ }^{3}\left(\frac{\partial^{2}}{\partial x^{2}} \omega\right) q \\
& +2 a\left(\frac{\partial^{3}}{\partial x^{3}} s_{1}\right) \pi-48 a\left(\frac{\partial}{\partial x} s_{1}\right) \operatorname{Er} N s_{0}^{2} \pi+60 a s_{0} q^{2}\left(\frac{\partial}{\partial x} \beta_{1}\right) \pi \\
& +54 a\left(\frac{\partial}{\partial x} \beta_{1}\right) \operatorname{Er}\left(\cos \left(\chi_{1}\right)\right)^{2} \pi+36 a\left(\frac{\partial}{\partial x} s_{1}\right) \operatorname{Er} N s_{0}{ }^{3} \pi-2 a\left(\frac{\partial^{3}}{\partial x^{3}} \beta_{1}\right) s_{0}{ }^{3} \pi \\
& -6\left(\frac{\partial^{3}}{\partial x^{3}} s_{1}\right) s_{0} \pi-6 a\left(\frac{\partial^{3}}{\partial x^{3}} \beta_{1}\right)\left(\cos \left(\chi_{1}\right)\right)^{2} s_{0}^{2} \pi+6 a\left(\frac{\partial}{\partial x} \beta_{1}\right) \operatorname{Er} N \pi \\
& +12 a s_{0}{ }^{3} q^{2}\left(\frac{\partial}{\partial x} \beta_{1}\right) \pi-30 a\left(\frac{\partial}{\partial x} \beta_{1}\right) \operatorname{Er} N s_{0} \pi+114 a\left(\frac{\partial}{\partial x} s_{1}\right) q^{2} s_{0}^{2} \pi \\
& -6 a\left(\frac{\partial^{3}}{\partial x^{3}} \beta_{1}\right) s_{0} \pi-6 a\left(\frac{\partial^{3}}{\partial x^{3}} \beta_{1}\right)\left(\cos \left(\chi_{1}\right)\right)^{2} \pi+4 a\left(\frac{\partial^{3}}{\partial x^{3}} s_{1}\right) s_{0}{ }^{3} \pi-6 a\left(\frac{\partial^{3}}{\partial x^{3}} s_{1}\right) s_{0}{ }^{2} \pi \\
& -18 a\left(\frac{\partial}{\partial x} \beta_{1}\right) \operatorname{Er} \pi-18 a\left(\frac{\partial}{\partial x} s_{1}\right) \operatorname{Er} \pi+6 a\left(\frac{\partial^{3}}{\partial x^{3}} \beta_{1}\right) s_{0}{ }^{2} \pi-444 a s_{0}{ }^{3} q^{3} \omega \\
& -24 a s_{0}\left(\frac{\partial^{2}}{\partial x^{2}} \omega\right) q+30 a s_{0}^{2}\left(\frac{\partial^{2}}{\partial x^{2}} \omega\right) q+60 a q^{3} s_{0} \omega+186 a q^{3} s_{0}^{2} \omega \\
& +12 a s_{0}{ }^{3}\left(\frac{\partial^{2}}{\partial x^{2}} \omega\right) q+144 a q^{3} s_{0}{ }^{4} \omega+2 a\left(\frac{\partial^{3}}{\partial x^{3}} \beta_{1}\right) \pi-90 s_{0}{ }^{2}\left(\frac{\partial^{2}}{\partial x^{2}} \omega\right) q \\
& -60 q^{2} a s_{0}\left(\frac{\partial}{\partial x} \beta_{1}\right)\left(\cos \left(\chi_{1}\right)\right)^{2} \pi-36 a\left(\frac{\partial}{\partial x} s_{1}\right) q^{2} s_{0}^{3} \pi+18 a\left(\frac{\partial}{\partial x} s_{1}\right) E r s_{0} \pi \\
& -36 a\left(\frac{\partial}{\partial x} \beta_{1}\right) \operatorname{Er} N\left(\cos \left(\chi_{1}\right)\right)^{2} s_{0}^{3} \pi+54 a\left(\frac{\partial}{\partial x} \beta_{1}\right) \operatorname{Er} N\left(\cos \left(\chi_{1}\right)\right)^{2} s_{0} \pi \\
& +6 a\left(\frac{\partial}{\partial x} s_{1}\right) \operatorname{Er} N s_{0} \pi-60 a\left(\frac{\partial}{\partial x} s_{1}\right) q^{2} s_{0} \pi+18 a\left(\frac{\partial}{\partial x} \beta_{1}\right) \operatorname{Er}_{0} \pi-54 a s_{0}^{2} q^{2}\left(\frac{\partial}{\partial x} \beta_{1}\right) \pi \\
& -6 a s_{0}^{2} q^{2}\left(\frac{\partial}{\partial x} \beta_{1}\right)\left(\cos \left(\chi_{1}\right)\right)^{2} \pi-54 a\left(\frac{\partial}{\partial x} \beta_{1}\right) \operatorname{Ers}_{0}\left(\cos \left(\chi_{1}\right)\right)^{2} \pi \\
& +12 a\left(\frac{\partial^{3}}{\partial x^{3}} \beta_{1}\right)\left(\cos \left(\chi_{1}\right)\right)^{2} s_{0} \pi+6 a\left(\frac{\partial}{\partial x} s_{1}\right) \operatorname{Er} N \pi+216 a s_{0} \omega \operatorname{Er} q \\
& +216 a s_{0}{ }^{3} \operatorname{Er} N \omega q-72 a s_{0} \omega \operatorname{Er} N q-144 a s_{0}{ }^{4} \operatorname{Er} N \omega q-216 a s_{0}{ }^{2} \omega \operatorname{Er} q+3\left(\frac{\partial^{3}}{\partial x^{3}} \beta_{1}\right) s_{0} \pi=0 .
\end{aligned}
$$




$$
\begin{aligned}
& 6 \alpha s_{0}^{3}\left(\frac{\partial^{3}}{\partial x^{3}} \varphi\right) \pi-6 \alpha s_{0}^{2}\left(\frac{\partial^{3}}{\partial x^{3}} \varphi\right) \pi+18\left(\frac{\partial^{2}}{\partial x^{2}} v_{y}\right) \operatorname{Er} \mu_{3} \pi+36\left(\frac{\partial^{2}}{\partial x^{2}} v_{y}\right) \operatorname{Er} \eta \pi \\
& -9 \mu_{2}\left(\frac{\partial^{2}}{\partial x^{2}} v_{y}\right) s_{0}{ }^{3} \operatorname{Er} \pi-36\left(\frac{\partial^{2}}{\partial x^{2}} v_{y}\right) \operatorname{Er} \eta s_{0} \pi+9 \mu_{2}\left(\frac{\partial^{2}}{\partial x^{2}} v_{y}\right) s_{0}{ }^{2} \operatorname{Er} \pi \\
& +12 \mu_{1}\left(\frac{\partial^{2}}{\partial x^{2}} v_{y}\right) s_{0} \operatorname{Er} \pi-18\left(\frac{\partial^{2}}{\partial x^{2}} v_{y}\right) \operatorname{Er} \mu_{3} s_{0} \pi \\
& -12 \mu_{1}\left(\frac{\partial^{2}}{\partial x^{2}} v_{y}\right) s_{0}{ }^{2} \operatorname{Er} \pi-48 a \alpha s_{0}{ }^{3} \operatorname{Er} N \beta_{1} \sin \left(\chi_{1}\right) \cos \left(\chi_{1}\right) q \\
& -8 a \alpha\left(\frac{\partial^{2}}{\partial x^{2}} \beta_{1}\right) \sin \left(\chi_{1}\right) \cos \left(\chi_{1}\right) s_{0}{ }^{2} q+4 a \alpha\left(\frac{\partial^{2}}{\partial x^{2}} \beta_{1}\right) \sin \left(\chi_{1}\right) \cos \left(\chi_{1}\right) q \\
& +72 a \alpha s_{0} E r N \beta_{1} \sin \left(\chi_{1}\right) \cos \left(\chi_{1}\right) q-24 a \alpha \beta_{1} \sin \left(\chi_{1}\right) \cos \left(\chi_{1}\right) \operatorname{Er} N q \\
& +22 a \alpha s_{0}\left(\frac{\partial^{2}}{\partial x^{2}} \beta_{1}\right) \sin \left(\chi_{1}\right) \cos \left(\chi_{1}\right) q-6 \alpha s_{0}\left(\frac{\partial^{2}}{\partial x^{2}} \beta_{1}\right) \sin \left(\chi_{1}\right) \cos \left(\chi_{1}\right) q \\
& +20 a \alpha q^{3} \beta_{1} \sin \left(\chi_{1}\right) \cos \left(\chi_{1}\right)-88 a \alpha q^{3} \beta_{1} \sin \left(\chi_{1}\right) \cos \left(\chi_{1}\right) s_{0}{ }^{2} \\
& -72 a \alpha \beta_{1} \sin \left(\chi_{1}\right) \cos \left(\chi_{1}\right) E r s_{0} q+24 \alpha q^{3} \beta_{1} \sin \left(\chi_{1}\right) \cos \left(\chi_{1}\right) s_{0}{ }^{2} \\
& +48 a \alpha q^{3} s_{0}{ }^{3} \beta_{1} \sin \left(\chi_{1}\right) \cos \left(\chi_{1}\right)+72 a \alpha \beta_{1} \sin \left(\chi_{1}\right) \cos \left(\chi_{1}\right) E r q \\
& -70 a \alpha q^{3} \beta_{1} \sin \left(\chi_{1}\right) \cos \left(\chi_{1}\right) s_{0}-42 \alpha q^{3} \beta_{1} \sin \left(\chi_{1}\right) \cos \left(\chi_{1}\right) s_{0}=0 .
\end{aligned}
$$

$$
\begin{aligned}
& 18\left(\frac{\partial^{2}}{\partial x^{2}} v_{z}\right) \operatorname{Er} \eta \pi+36 a \alpha\left(\frac{\partial}{\partial x} \beta_{1}\right) \sin \left(\chi_{1}\right) \cos \left(\chi_{1}\right) \operatorname{Er} N s_{0} \\
& +36 a \alpha\left(\frac{\partial}{\partial x} \beta_{1}\right) \sin \left(\chi_{1}\right) \cos \left(\chi_{1}\right) \operatorname{Er}-24 a \alpha\left(\frac{\partial}{\partial x} \beta_{1}\right) \operatorname{ErN} s_{0}{ }^{3} \sin \left(\chi_{1}\right) \cos \left(\chi_{1}\right) \\
& -4 a \alpha\left(\frac{\partial^{3}}{\partial x^{3}} \beta_{1}\right) \sin \left(\chi_{1}\right) \cos \left(\chi_{1}\right)+57 \alpha\left(\frac{\partial}{\partial x} \beta_{1}\right) q^{2} \sin \left(\chi_{1}\right) \cos \left(\chi_{1}\right) s_{0} \\
& -2 a \alpha\left(\frac{\partial}{\partial x} \beta_{1}\right) q^{2} \sin \left(\chi_{1}\right) \cos \left(\chi_{1}\right)+8 a \alpha\left(\frac{\partial^{3}}{\partial x^{3}} \beta_{1}\right) \sin \left(\chi_{1}\right) \cos \left(\chi_{1}\right) s_{0} \\
& -4 a \alpha\left(\frac{\partial^{3}}{\partial x^{3}} \beta_{1}\right) \sin \left(\chi_{1}\right) \cos \left(\chi_{1}\right) s_{0}{ }^{2}-12 \alpha\left(\frac{\partial}{\partial x} \beta_{1}\right) q^{2} \sin \left(\chi_{1}\right) \cos \left(\chi_{1}\right) s_{0}{ }^{2} \\
& -12 a \alpha\left(\frac{\partial}{\partial x} \beta_{1}\right) \sin \left(\chi_{1}\right) \cos \left(\chi_{1}\right) \operatorname{Er} N-41 a \alpha\left(\frac{\partial}{\partial x} \beta_{1}\right) q^{2} \sin \left(\chi_{1}\right) \cos \left(\chi_{1}\right) s_{0} \\
& -44 a \alpha\left(\frac{\partial}{\partial x} \beta_{1}\right) q^{2} \sin \left(\chi_{1}\right) \cos \left(\chi_{1}\right) s_{0}^{2}+24 a \alpha s_{0}{ }^{3} q^{2}\left(\frac{\partial}{\partial x} \beta_{1}\right) \sin \left(\chi_{1}\right) \cos \left(\chi_{1}\right) \\
& -36 a \alpha\left(\frac{\partial}{\partial x} \beta_{1}\right) \sin \left(\chi_{1}\right) \cos \left(\chi_{1}\right) \operatorname{Er} s_{0}-9\left(\frac{\partial^{2}}{\partial x^{2}} v_{z}\right) \operatorname{Er} \mu_{3} s_{0} \pi \\
& +6 \alpha s_{0}^{3} q\left(\frac{\partial^{2}}{\partial x^{2}} \varphi\right) \pi-18\left(\frac{\partial^{2}}{\partial x^{2}} v_{z}\right) \operatorname{Er} \eta s_{0} \pi+3 \mu_{1}\left(\frac{\partial^{2}}{\partial x^{2}} v_{z}\right) s_{0}{ }^{2} \operatorname{Er} \pi-18 \operatorname{Er} \pi \\
& -3 \mu_{1}\left(\frac{\partial^{2}}{\partial x^{2}} v_{z}\right) s_{0} \operatorname{Er} \pi+18 \operatorname{Er} s_{0} \pi-6 \alpha{s_{0}}^{2} q\left(\frac{\partial^{2}}{\partial x^{2}} \varphi\right) \pi+9\left(\frac{\partial^{2}}{\partial x^{2}} v_{z}\right) \operatorname{Er} \mu_{3} \pi=0 .
\end{aligned}
$$




\section{Appendix B. The coefficients in the solutions.}

$$
\begin{aligned}
C_{1}= & -\frac{q}{2 D_{1}}\left(-q^{4} \sinh (r) \cos (s) k_{2}{ }^{2}-\sinh (r) \cos (s) \omega^{2}+k_{1} k_{2} q^{2} \sinh (r) \cos (s) r^{2}\right. \\
& \left.-2 k_{1} k_{2} q^{2} \cosh (r) r \sin (s) s-k_{1} k_{2} q^{2} \sinh (r) \cos (s) s^{2}\right) ; \\
C_{2}= & \frac{q}{2 D_{1}}\left(-\cosh (r) \sin (s) q^{4}{k_{2}}^{2}-k_{2} q^{2} \cosh (r) \sin (s) k_{1} s^{2}+k_{2} q^{2} \cosh (r) \sin (s) k_{1} r^{2}\right. \\
& \left.+2 k_{2} q^{2} k_{1} r s \sinh (r) \cos (s)-\cosh (r) \sin (s) \omega^{2}\right) ; \\
C_{3}= & -\frac{q}{D_{2}}\left(A_{1} \sinh (r) r^{2} k_{1} k_{2} q^{2} \cosh (r) s-k_{1} s \sinh (r) \omega^{2} \cosh (r)\right. \\
& -k_{1} s \cosh (r) q^{4} k_{2}{ }^{2} \sinh (r)+A_{1} \sinh (r) s \cosh (r) q^{4} k_{2}{ }^{2} \\
& +A_{1} \sinh (r) s^{3} k_{2} q^{2} \cosh (r) k_{1}-k_{1}{ }^{3} k_{2} q^{2} \cosh (r) \sinh (r) \\
& +A_{1} \cosh (r) s \sinh (r) \omega^{2}-k_{1}{ }^{2} r^{3} k_{2} q^{2} \sin (s) \cos (s)+A_{1} \cos (s) r^{3} k_{2} q^{2} \sin (s) k_{1} \\
& -k_{1}{ }^{2} r k_{2} q^{2} \sin (s) s^{2} \cos (s)-2 A_{1}(\cosh (r))^{2} k_{1} k_{2} q^{2} r s-A_{1} \sin (s) r \cos (s) \omega^{2} \\
& -A_{1} \cos (s) r \sin (s) q^{4} k_{2}{ }^{2}+A_{1} \cos (s) r k_{2} q^{2} \sin (s) k_{1} s^{2}+k_{1} r \cos (s) \omega^{2} \sin (s) \\
& \left.+k_{1} r \sin (s) q^{4} k_{2}{ }^{2} \cos (s)+2 A_{1}(\cos (s))^{2} k_{2} q^{2} k_{1} r s-k_{1}{ }^{2} s k_{2} q^{2} \cosh (r) r^{2} \sinh (r)\right)
\end{aligned}
$$

$$
\begin{aligned}
& C_{4}=-\frac{q}{\omega\left(r^{2}+s^{2}\right) D_{2}}\left(2 A_{1} r^{2} s^{3} \cosh (r) k_{1} \sinh (r) \omega^{2}-2 k_{2}^{2} q^{4} r^{3} \cos (s) k_{1}^{2} \sin (s) s^{2}\right. \\
& -A_{1} k_{2} q^{2} s^{3} \cosh (r) \sinh (r) \omega^{2}-2 k_{2} q^{2} s^{3} \cosh (r) k_{1} \sinh (r) \omega^{2} \\
& -2 k_{2}{ }^{2} q^{4} \cosh (r) s \sinh (r) \omega^{2}-2 A_{1} k_{2}{ }^{2} q^{4} r^{5} \cos (s) \sin (s) k_{1}-k_{2}{ }^{2} q^{4} r^{5} \cos (s) k_{1}{ }^{2} \sin (s) \\
& -A_{1} s^{5} \cosh (r) k_{1} \sinh (r) \omega^{2}+2 A_{1} r^{3} s^{2} \sin (s) k_{1} \cos (s) \omega^{2} \\
& +A_{1} k_{2} q^{2} s^{2} \sin (s) r \cos (s) \omega^{2}+2 \omega^{2} \cosh (r) r^{2} k_{2} q^{2} k_{1} s \sinh (r) \\
& -A_{1} k_{2} q^{2} r^{2} \cosh (r) s \sinh (r) \omega^{2}+2 k_{2}^{3} q^{6} \cos (s) r^{3} \sin (s) k_{1} \\
& +A_{1} r^{7} \cos (s) k_{1}{ }^{2} k_{2} q^{2} \sin (s)+2 A_{1} k_{2}{ }^{2} q^{4} s^{4} \cos (s) r \sin (s) k_{1} \\
& +A_{1} k_{2} q^{2} r^{3} \sin (s) \cos (s) \omega^{2}+2 A_{1} r^{3} k_{1} s(\cos (s))^{2} \omega^{2}-2 A_{1} r^{3} k_{1} s(\cosh (r))^{2} \omega^{2} \\
& +2 A_{1} s^{3} k_{1} r(\cos (s))^{2} \omega^{2}-2 A_{1} s^{3} k_{1} r(\cosh (r))^{2} \omega^{2}-\omega^{4} \sin (s) r \cos (s) \\
& +2 \omega^{2} \sin (s) r^{3} k_{1} k_{2} q^{2} \cos (s)+3 A_{1} s^{4} \sin (s) k_{1} r \cos (s) \omega^{2}-2 \omega^{2} \sin (s) r q^{4} \cos (s) k_{2}{ }^{2} \\
& -2 \omega^{2} \sin (s) s^{2} k_{2} q^{2} k_{1} r \cos (s)-k_{2}{ }^{4} q^{8} \cos (s) r \sin (s)-2 k_{2}{ }^{3} q^{6} \cos (s) r \sin (s) k_{1} s^{2} \\
& -k_{2}{ }^{2} q^{4} s^{4} \cos (s) k_{1}{ }^{2} r \sin (s)+A_{1} s^{6} \cos (s) k_{1}{ }^{2} k_{2} q^{2} r \sin (s) \\
& -A_{1} k_{2}{ }^{3} q^{6} s^{3} \sinh (r) \cosh (r)+A_{1} k_{2}{ }^{3} q^{6} r^{3} \cos (s) \sin (s)-k_{2}{ }^{4} q^{8} \sinh (r) s \cosh (r) \\
& -k_{2}{ }^{2} q^{4} s^{5} \sinh (r) k_{1}{ }^{2} \cosh (r)+2 A_{1} r^{4} \sinh (r) k_{1} s \cosh (r) q^{4} k_{2}{ }^{2} \\
& -2 k_{2}{ }^{2} q^{4} r^{2} \sinh (r) k_{1}{ }^{2} s^{3} \cosh (r)-A_{1} k_{2}{ }^{3} q^{6} r^{2} \sinh (r) s \cosh (r) \\
& -3 A_{1} r^{4} \sinh (r) k_{1}^{2} s^{3} k_{2} q^{2} \cosh (r)-2 A_{1} s^{5} \sinh (r) k_{1} \cosh (r) q^{4} k_{2}^{2} \\
& +2 k_{2}{ }^{3} q^{6} \sinh (r) r^{2} k_{1} \cosh (r) s \\
& -3 A_{1} s^{5} \sinh (r) k_{1}{ }^{2} k_{2} q^{2} \cosh (r) r^{2}-2 k_{2}{ }^{3} q^{6} \sinh (r) s^{3} \cosh (r) k_{1} \\
& -\omega^{4} \cosh (r) s \sinh (r)-A_{1} r^{6} \sinh (r) k_{1}{ }^{2} k_{2} q^{2} \cosh (r) s \\
& -A_{1} s^{7} \sinh (r) k_{1}{ }^{2} k_{2} q^{2} \cosh (r)-k_{2}{ }^{2} q^{4} r^{4} \sinh (r) k_{1}{ }^{2} s \cosh (r) \\
& +3 A_{1} r^{3} s^{4} \cos (s) k_{1}{ }^{2} k_{2} q^{2} \sin (s)+3 A_{1} r^{5} s^{2} \cos (s) k_{1}{ }^{2} k_{2} q^{2} \sin (s) \\
& +3 A_{1} r^{4} \cosh (r) k_{1} s \sinh (r) \omega^{2}-A_{1} r^{5} \sin (s) k_{1} \cos (s) \omega^{2} \\
& \left.+A_{1} k_{2}{ }^{3} q^{6} s^{2} \cos (s) r \sin (s)\right) \text {; }
\end{aligned}
$$




$$
\begin{aligned}
& D_{1}=k_{1} r s\left((\sinh (r))^{2}(\cos (s))^{2}+(\cosh (r))^{2}(\sin (s))^{2}\right)\left(k_{2}^{2} q^{4}+\omega^{2}\right) ; \\
& D_{2}=k_{1} r s\left((\cos (s))^{2}-(\cosh (r))^{2}\right)\left(k_{2}^{2} q^{4}+\omega^{2}\right) .
\end{aligned}
$$

\section{REFERENCES}

[1] B. Bird, R.C. Armstrong and O. Hassager, Dynamics of Polymeric Liquids, 1-2, John Wiley and Sons, New York, 1987.

[2] Burghardt WR, Oscillatory shear flow of nematic liquid crystals, J. Rheol., 35(1), 49-62, 1991.

[3] M.C. Calderer, M.G. Forest and Q. Wang, Kinetic Theories and Mesoscopic models for solutions of nonhomogeneous liquid crystal polymers, J. Non-Newtonian Fluid Mechanics, 120, 69-78, 2004.

[4] Eric Choate, Zhenlu Cui and M. Gregory Forest, Effects of strong anchoring on the dynamics moduli of heterogeneous nematic polymers, Rheological Acta., 47, 223-236, 2008.

[5] Chandrasekhar, Liquid Crystals, 2nd ed. Cambridge University Press, Cambridge, 1992.

[6] Z. Cui, M.C. Calderer and Q. Wang, Mesostructures in flows of weakly sheared chiral liquid crystalline polymers, Discrete and Continuous Dynamical Systems-Series B, 6(2), 291-310, 2006.

[7] Z. Cui, Q. Wang, A continuum model for flows of chiral liquid crystal polymers and permeation flows, J. Non-Newtonian Fluid Mech., 138, 44-61, 2006.

[8] Z. Cui and Q. Wang, Cholesteric liquid crystal polymers under permeation oscillatory shear, submitted.

[9] de Andrade Lima LRP, Rey AD, Superposition and universality in the linear viscoelasticity of Leslie-Ericken liquid crystals, J. Rheol., 48, 1067-1084, 2004.

[10] de Andrade Lima LRP, Rey AD, Assessing flow alignment of nematic liquid crystals through linear viscoelasticity, Phys. Rev. E, 70, 011701, 2004.

[11] de Andrade Lima LRP, Rey AD, Superposition principles for small amplitude oscillatory shearing of nematic mesophases, Rheol. Acta, 45, 591-600, 2006.

[12] P.G. de Gennes and J. Prost, The Physics of Liquid Crystals, Oxford University Press, 1993.

[13] G. Derfel, Shear flow induced cholesteric-nematic transition, Mol. Cryst. Liq. Cryst., 92, 41-47, 1983.

[14] W. Helfrich, Capillary flow of cholesteric and semctic liquid crystals, Phys. Rev. Lett., 23, 372, 1969.

[15] W. Helfrich, Capillary viscometry of cholesteric liquid crystals, Proceedings Amer. Chem. Soc., 2nd ed., 1969, 405-418, 1970.

[16] U.D. Kini, G.S. Ranganath and S. Chandrasekhar, Flow of cholesteric liquid crystals-I: Flow along the helical axis, Pramana, 5, 101-106, 1975.

[17] U.D. Kini, Shear flow of cholesterics normal to the helical axis, J. Phys. (France), 40, 62-65, 1979.

[18] R.G. Larson, The Structure and Rheology of Complex Fluids, Oxford University Press, 1999.

[19] F.M. Leslie, Continuum theory of cholesteric liquid crystals, Mol. Cryst. Liq. Cryst., 7, 407-420, 1969.

[20] T. Lubenski, Hydrodynamics of cholesteric liquid crystals, Phys. Rev. A, 6, 452-461, 1969.

[21] D. Marenduzzo, E. Orlandini and J.M. Yeomans, Permeative flows in cholesteric liquid crystals, Physical Review Letters, V. 92(18), 188301-1-188301-4, 2004.

[22] D. Marenduzzo, E. Rlandini and J.M. Yeomans, Interplay between shear flow and elastic deformations in liquid crystals, J. Chem. Phys., 121 (1), 582-591, 2004.

[23] J. Prost, Y. Pomeau and E. Guyon, Stability of permeative flows in 1 dimensionally ordered systems, J. Phys. II, 1, 289-309, 1991.

[24] A.D. Rey, Flow-alignment in the helix uncoiling of cholesteric liquid crystals, Phys. Rev. E, 53, 4198-4201, 1996.

[25] A.D. Rey, Structural transformations and viscoelastic response of shear fingerprint cholesteric textures, J. Non-Newt. Fluid Mech., 64, 207-227, 1996.

[26] A.D. Rey, Helix uncoiling modes of sheared cholesteric liquid crystals, J. Chem. Phys., 104, 789-792, 1996.

[27] A.D. Rey, Theory of linear viscoelasticity for chiral liquid crystals, Rheol. Acta, 35, 400-409, 1996.

[28] A.D. Rey, Theory of linear viscoelasticity in cholesteric liquid crystals, J. Rheol., 44, 855-869, 2000.

[29] A.D. Rey, Generalized cholesteric permeation flows, Phys. Rev. E, 65, 022701, 2002.

[30] A.D. Rey, Simple shear and small amplitude oscillatory rectilinear shear permeation flows of cholesteric liquid crystals, J. Rheol., 46(1), 225-240, 2002. 
[31] N. Scaramuzza, F. Simoni and R. Bartolino, Permeative flow in cholesteric liquid crystals, Phys. Rev. Lett., 53(23), 2246-2249, 1984.

[32] Q. Wang, Biaxial steady states and their stability in shear flows of liquid crystal polymers, J. Rheol., 41(5), 943-970, 1997.

[33] Q. Wang, A hydrodynamic theory of nematic liquid crystalline polymers of different configurations, J. Chem. Phys., 116(20), 9120-9136, 2002. 